Revue des patrimoines

17 | 2011

Les patrimoines de l'enseignement supérieur

\title{
La constitution du domaine de la Cité internationale universitaire de Paris
}

Brigitte Blanc

\section{(2) OpenEdition}

Journals

Édition électronique

URL : http://journals.openedition.org/insitu/855

DOI : $10.4000 /$ insitu. 855

ISSN : 1630-7305

Éditeur

Ministère de la culture

Référence électronique

Brigitte Blanc, « La constitution du domaine de la Cité internationale universitaire de Paris », In Situ [En ligne], 17 | 2011, mis en ligne le 24 novembre 2011, consulté le 19 avril 2019. URL : http:// journals.openedition.org/insitu/855; DOI : 10.4000/insitu.855

Ce document a été généré automatiquement le 19 avril 2019

\section{(c) $($ i) $(9)$}

In Situ Revues des patrimoines est mis à disposition selon les termes de la licence Creative Commons Attribution - Pas d'Utilisation Commerciale - Pas de Modification 4.0 International. 


\title{
La constitution du domaine de la Cité internationale universitaire de Paris
}

\author{
Brigitte Blanc
}

1 Construite à partir des années 1920 à l'emplacement de l'enceinte de Thiers, la Cité internationale universitaire de Paris est conçue pour offrir des logements «salubres » à quelques milliers de jeune gens venus de tous les pays $d u$ monde. Initiative philanthropique destinée à faciliter la vie des étudiants, c'est aussi une œuvre à caractère éducatif voulant contribuer à la paix entre les nations; ses fondateurs assignent à ce "monde en miniature» un rôle de formation civique de la jeunesse. Plus de 5500 étudiants cohabitent étroitement, pour la compréhension et l'amitié, dans une communauté regroupant plus de 130 nationalités ${ }^{1}$.

2 Son implantation après la première guerre mondiale s'inscrit comme un des moments forts de l'histoire du sud parisien. Participant à la grande opération de transformation des fortifications qui vise à aérer la capitale, un nouveau quartier voit le jour entre le parc Montsouris et les communes de Gentilly et de Montrouge. 38 "maisons » sont aujourd'hui réparties sur un terrain verdoyant de 34 ha (fig. $\left.\mathbf{n}^{\circ} \mathbf{1}\right)$. S'ajoutant au noyau initial de 29 ha, de nouveaux terrains acquis ou concédés portent le domaine à plus de 40 ha - son extension maximale au début des années cinquante - avant la création du boulevard périphérique qui l'ampute d'une large bande au sud. Cet ensemble immobilier (dont près de la moitié est réservée à l'habitation) se compose de terrains d'origines diverses, parmi lesquels on distingue l'ancienne enceinte fortifiée, la zone non aedificandi aménagée en parc, des terrains détachés de la commune de Gentilly et, isolée de ce vaste quadrilatère d'un seul tenant, une parcelle située à l'angle du boulevard Jourdan et de la rue de la Tombe Issoire. Le statut juridique de ces différents espaces n'est pas homogène : certains sont propriété de l'Université de Paris, établissement public national, qui est aussi locataire de terrains communaux appartenant à la Ville de Paris et affectataire de biens domaniaux mis à sa disposition par l'État. La situation relativement claire lors de la création de la Cité se trouve compliquée à deux reprises par d'importants échanges de 
terrains. Ces remaniements et la difficulté relative à l'identification des parcelles intéressées par les divers projets ne permettent pas d'en établir l'origine avec une certitude absolue.

Figure 1

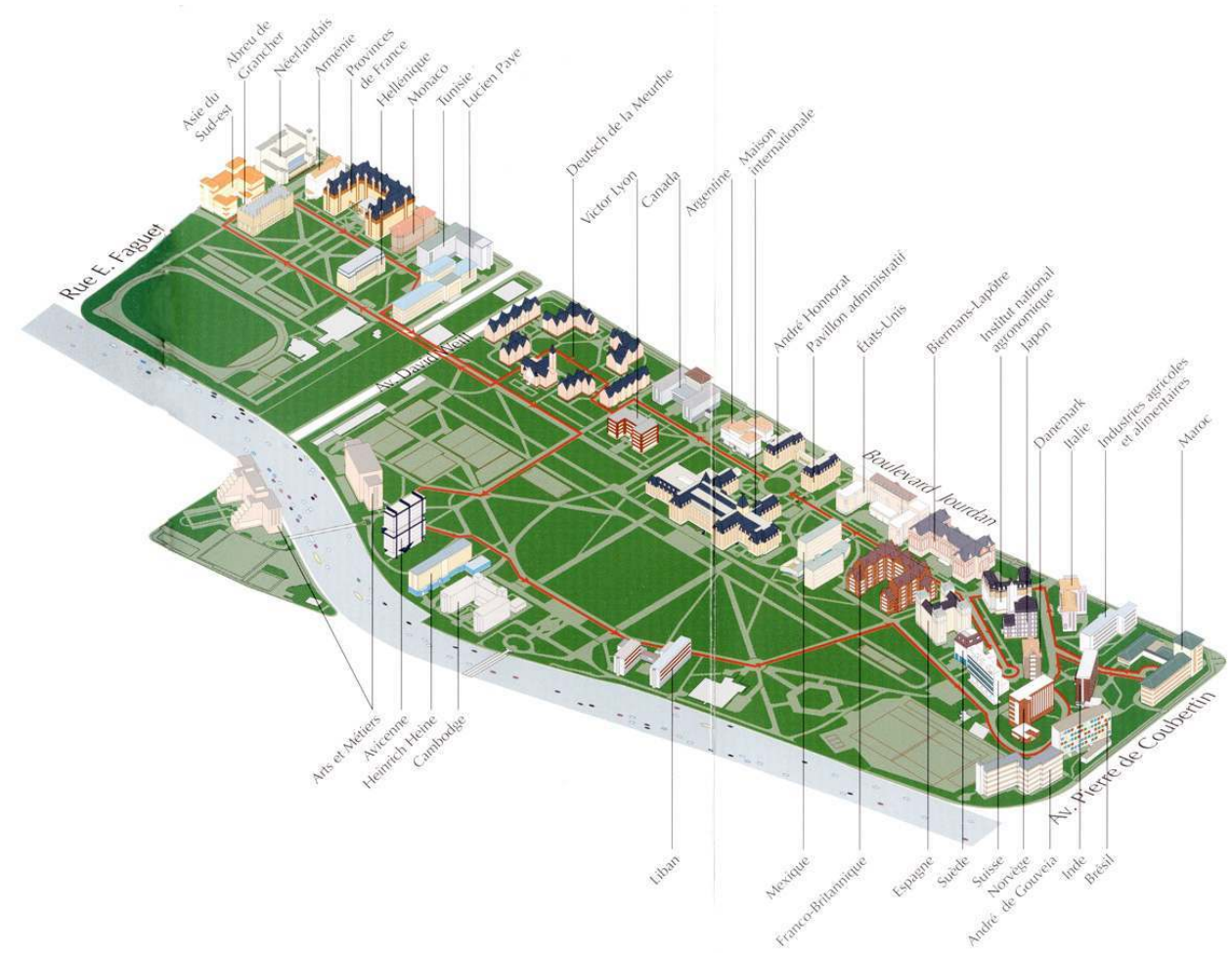

La Cité internationale universitaire de Paris : plan d'ensemble ; publié dans Cité internationale universitaire de Paris, Guide de visite, s.d.

(c) Droits réservés.

\section{À l'origine de la Cité : la convention de 1921}

La fondation de la Cité universitaire résulte d'une convention intervenue le 7 juin 1921 entre l'Université et la Ville de Paris. À partir de la donation d'Émile Deutsch de la Meurthe qui affecte un capital de 10 millions de $\mathrm{F}$ à la construction d'un «hameaujardin » au profit de 350 étudiants peu fortunés, se réalise un projet qu'André Honnorat, alors ministre de l'Instruction publique, a déjà eu l'occasion d'ébaucher lors des débats parlementaires de 1919 sur le déclassement de l'enceinte fortifiée de Paris. Accueillant avec enthousiasme l'offre du donateur, le ministre de l'Instruction publique y voit le point de départ d'un vaste ensemble international qui permettrait de consolider le rayonnement mondial de l'université de Paris tout en donnant aux futures élites les moyens de se connaitre et de fraterniser. Un site est trouvé en bordure du parc Montsouris, sur les bastions $n^{\circ} 81,82$ et 83 des anciennes fortifications, qui présentent un développement d'environ $900 \mathrm{~m} \times 100 \mathrm{~m}$ de profondeur entre la rue de la Tombe-Issoire et la porte de Gentilly (fig. $\mathbf{n}^{\circ} \mathbf{2}$ ). 


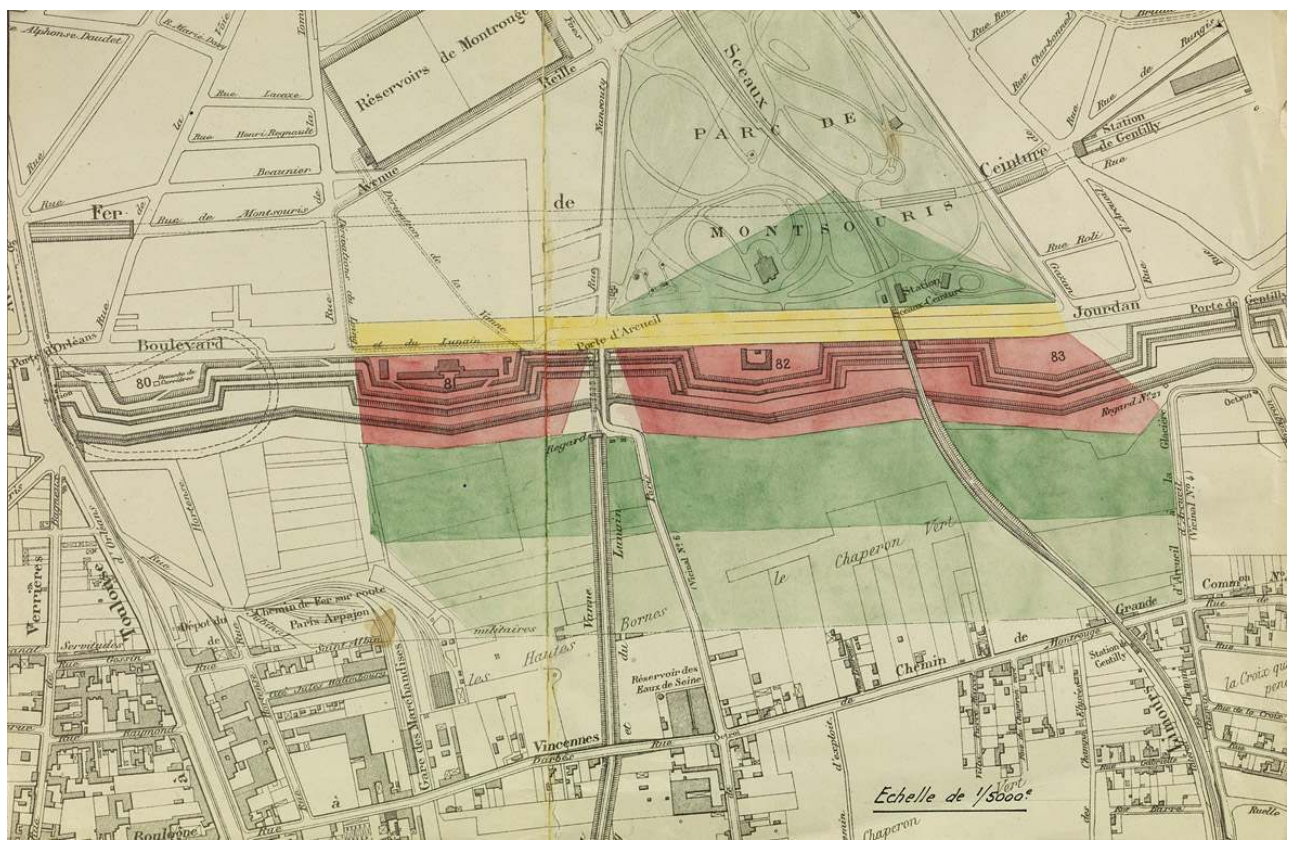

Plan des bastions 81, 82, 83, entre la Porte de Gentilly et la rue de la Tombe Issoire, cédés par la Ville de Paris à l'État pour la création de la Cité universitaire, 1921. AN, AJ16 /7034. Repro. Ayrault, Philippe.

(c) Conseil régional d'Île-de-France, service Patrimoines et Inventaire, 2009. [Base Mémoire : 20097501734NUC4A]

Par convention du 7 juin 1921, confirmée le 28 par le vote d'une loi, la Ville de Paris cède ainsi à l'État, pour la création d'une cité universitaire, une bande de terrains à bâtir de 9 ha environ parallèle au boulevard Jourdan, c'est-à-dire le sol des trois bastions, moyennant le versement d'une somme de 13 millions et demi payable en quinze annuités de $900000 \mathrm{~F}$ dont la première doit être versée le $1^{\mathrm{er}}$ mai 1923. La Ville prend à sa charge la remise en état du terrain, l'établissement des voies d'accès, et met à la disposition de la Cité la zone de servitude non aedificandi, après expropriation à ses frais des occupants et aménagement d'un parc avant la fin de l'année 1924: la convention prévoit en effet la remise à l'Université des terrains de la zone extérieure qui s'étendent au sud des précédents, moyennant une redevance annuelle de 1 franc par hectare et par an (fig. $\mathbf{n}^{\circ} \mathbf{3}$ ) . Cette redevance porte au total sur 20,65 ha à maintenir en espaces libres à usage de parc et de terrains de jeux. Le domaine initial ainsi constitué comprend donc un peu plus de 29 ha, bordés à l'ouest par la rue Émile-Faguet, à l'est par les rues Alexandre-de-Humboldt et Benoît-Malon, au nord par le boulevard Jourdan et au sud par une " voie d'isolement " qui sépare la zone de la commune de Gentilly (fig. $\left.\mathbf{n}^{\circ} \mathbf{4}\right)$. 


\section{Figure 3}

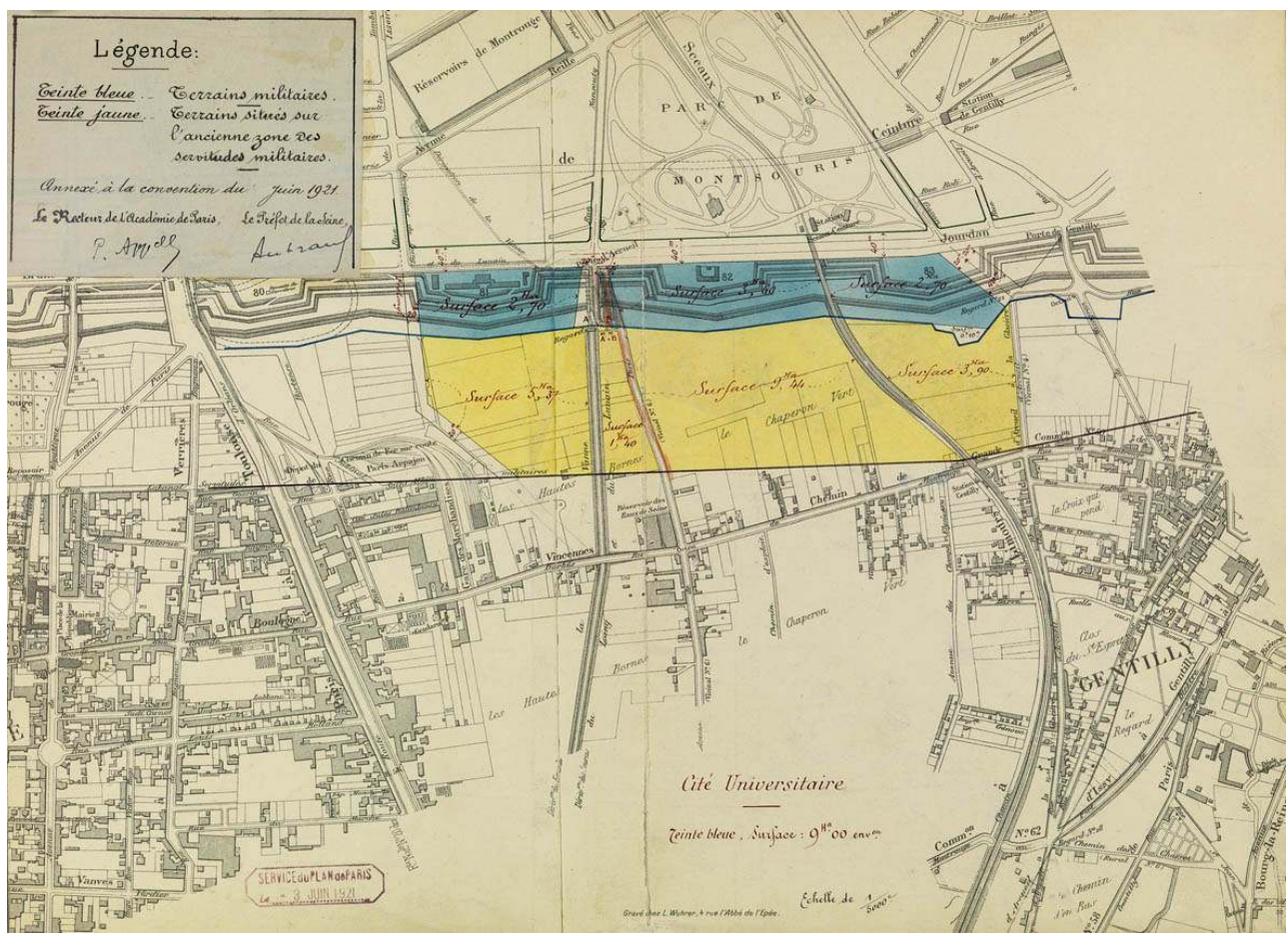

L'emprise de la Cité universitaire sur l'enceinte fortifiée (bastions 81-83) et les terrains de la zone de servitude non aedificandi, plan annexé à la convention du 7 juin 1921. AN, AJ16 /7027. Repro. Ayrault, Philippe.

(c) Conseil régional d'île-de-France, service Patrimoines et Inventaire, 2009. [Base Mémoire : 20097501742NUC4A]

\section{Figure 4}

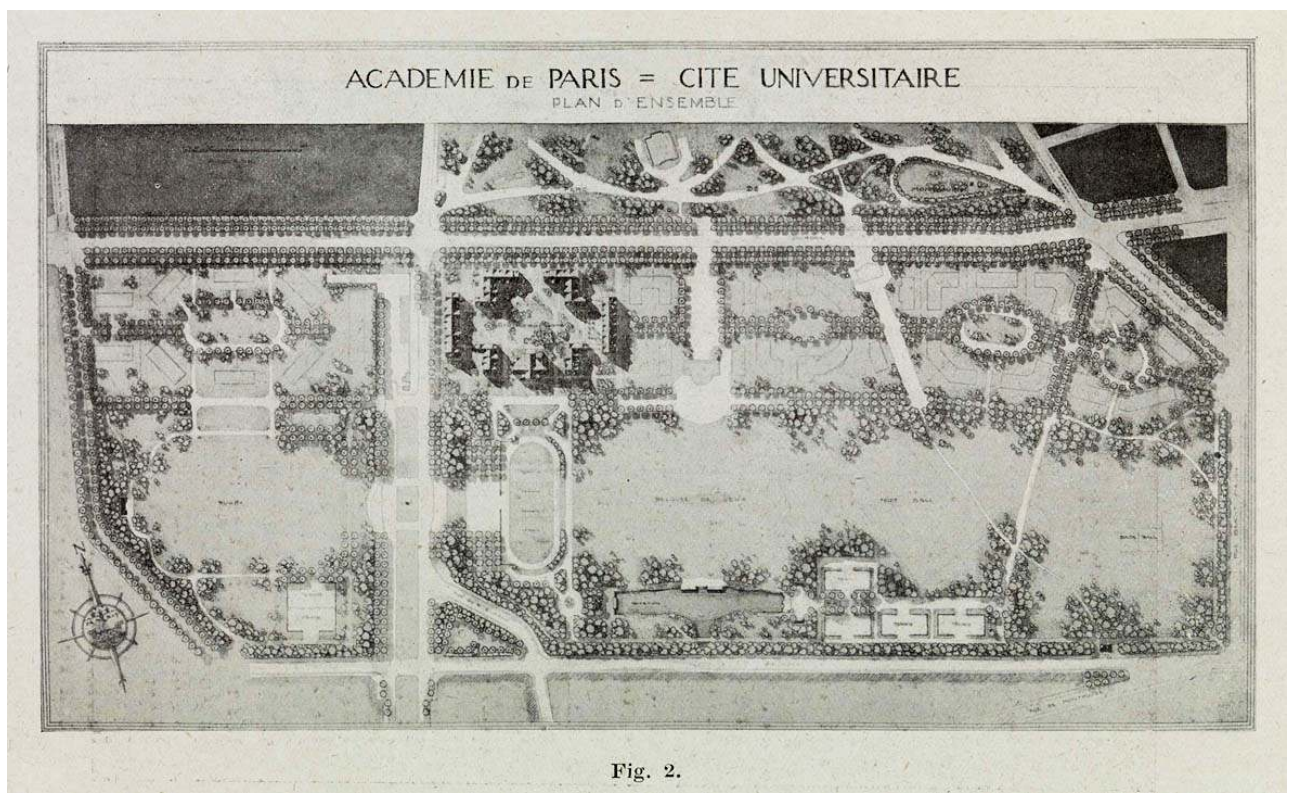

Plan d'ensemble représentant le site initial de la Cité avec le dessin du parc et la Fondation Deutsch de la Meurthe, dans BECHMANN, L. La Cité universitaire de Paris, 1923. AN, AJ16 /7027. Repro. Ayrault, Philippe.

(c) Conseil régional d'île-de-France, service Patrimoines et Inventaire, 2009. [Base Mémoire : 20097501732NUC4A] 


\section{L'extension du domaine, 1928-1941}

\section{À l'est des bastions d'origine : l'« îlot 19 du XIVe arrondissement "}

Suivant l'exemple d'Émile Deutsch de la Meurthe et répondant à l'appel des fondateurs, des personnalités ou des gouvernements étrangers prennent des initiatives en faveur de la construction des premières résidences. Dès le début de 1927 - soit quatre ans après la pose de la première pierre de la fondation Deutsch (fig. $\mathbf{n}^{\circ} 5$ ) - la moitié du domaine est déjà lotie : les fondations achevées (Deutsch de la Meurthe, Canada, Argentine, Belgique, Angleterre, Japon) ou en cours de construction (Institut agronomique), qui logeront 1200 étudiants à partir de 1929, occupent près de 4 ha sur 9 (dont plus d'un réservé à l'emprise du chemin de fer de la ligne de Sceaux et du double aqueduc de la Vanne et du Loing, ainsi qu'à l'aménagement des allées ${ }^{2}$ ). Or la Fondation nationale ${ }^{3}$ est saisie de multiples demandes - États-Unis, Italie, Espagne, Suède, Hollande, Tchécoslovaquie, Grèce, Brésil, Colombie et Venezuela - sans compter les projets élaborés pour les étudiants français - auxquelles il lui parait impossible de donner suite si le domaine n'est pas immédiatement agrandi.

Figure 5

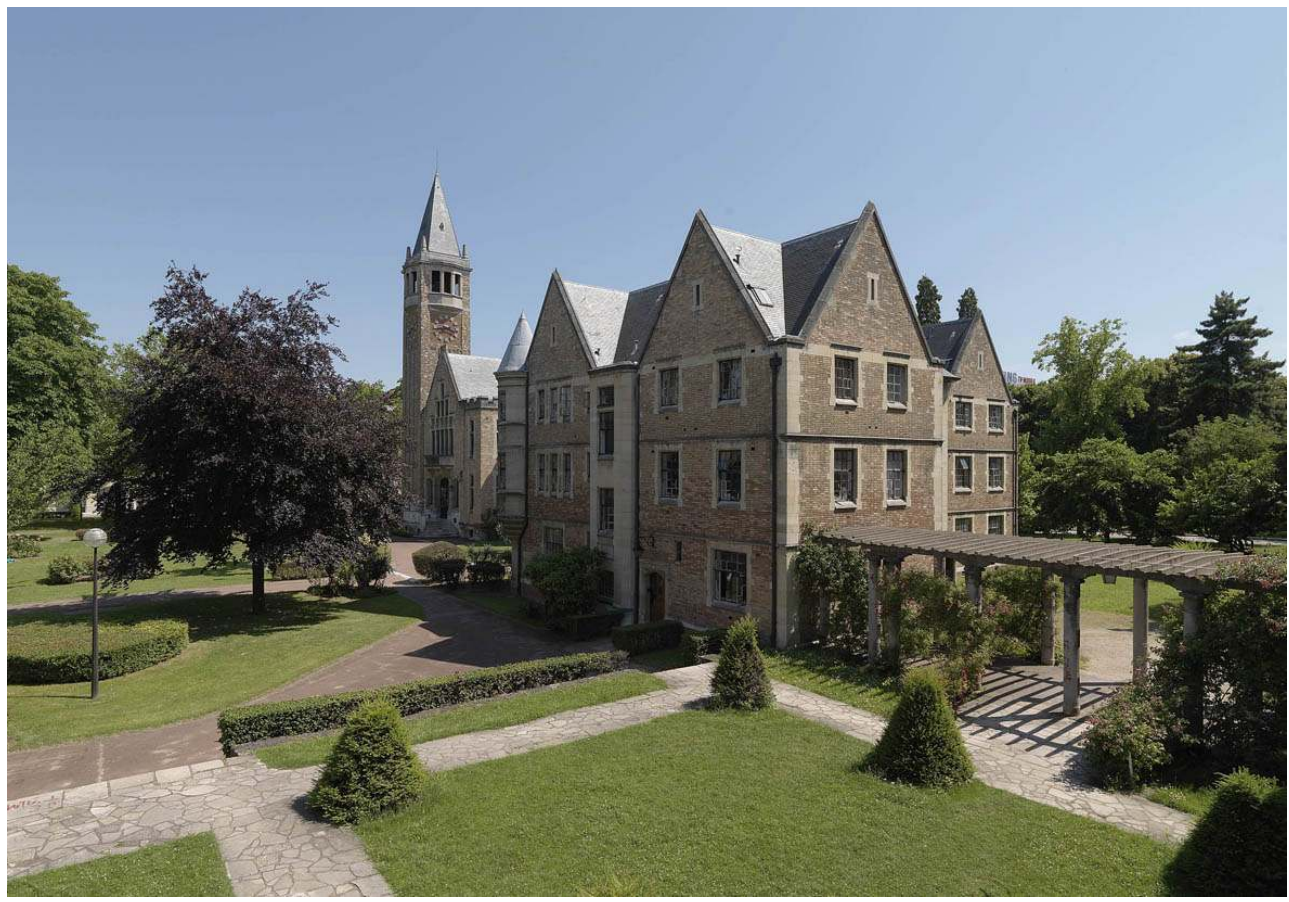

La Fondation Émile et Louise Deutsch de la Meurthe : le pavillon central flanqué d'un beffroi et le pavillon Poincaré. Phot. Ayrault, Philippe.

(c) Conseil régional d'île-de-France, service Patrimoines et Inventaire, 2009. [Base Mémoire: 20097501149NUC4A]

Une opportunité est alors fournie par une libéralité de David David-Weill, associé gérant de la Banque Lazare, philanthrope et mécène ${ }^{4}$, qui met à la disposition de l'Université de Paris une somme de 1950000 F pour l'acquisition de l'« îlot 19 du XIV arrondissement », parcelle d'1,31 ha située sur l'ancien bastion 84, entre la rue Alexandre-de-Humboldt et la porte de Gentilly (fig. $\mathbf{n}^{\circ} \mathbf{6}$ ). Le 8 juillet 1927 , le conseil municipal autorise cette cession 
(approuvée par décret présidentiel du 29 avril 1928), mais diffère sa décision sur les terrains de zone adjacents que la Fondation nationale souhaite incorporer au parc de la Cité. La remise de l'îlot est subordonnée à la signature d'une convention qui, le 28 février 1929, fixe les conditions de paiement ( 15 annuités de $130000 \mathrm{~F}$ chacune), sans consentir à la location des terrains zoniers situés au droit de ce lot. La Ville et la préfecture de la Seine opposent une vive résistance aux multiples démarches du comité de direction: cette partie de la zone reste en effet, dans le quartier de la Santé, le seul terrain susceptible d'être ouvert au public ${ }^{5}$, le stade Fémina, le dépôt d'Arpajon, les cimetières de Montrouge et de Gentilly occupant déjà sur la zone des surfaces considérables au détriment des promenades publiques et des terrains de jeux. En 1934, la Fondation nationale avance un nouvel argument: d'après le programme adopté par le comité de direction, ce secteur doit constituer le quartier de la Cité universitaire spécialement réservé aux étudiantes ${ }^{6}$ et comprendre les espaces nécessaires à l'aménagement de tennis et jardins affectés à leur usage exclusif (fig. $\mathbf{n}^{\circ} \mathbf{7}$ ). Elle accepte la transaction proposée le 28 juin 1933 par les services de la préfecture ${ }^{7}$, consistant à agrandir le «terrain DavidWeill $»^{8}$ par la suppression de la rue Nisard adjacente et l'adjonction d'un lot prélevé sur la partie voisine de la zone - le tout mesurant environ $8300 \mathrm{~m}^{2}$-, à condition qu'en échange la Cité rende à la Ville de Paris le terrain nécessaire pour articuler un large pan coupé à l'angle de l'avenue de la Porte de Gentilly et du boulevard Jourdan. Mais les pourparlers sont momentanément suspendus et n'aboutiront qu'en 1941 et 1944 dans le cadre des échanges de terrains induits par l'extension de la Cité sur le territoire de la commune Gentilly puis l'élargissement à $50 \mathrm{~m}$ de la rue de Montrouge.

\section{Figure 6}

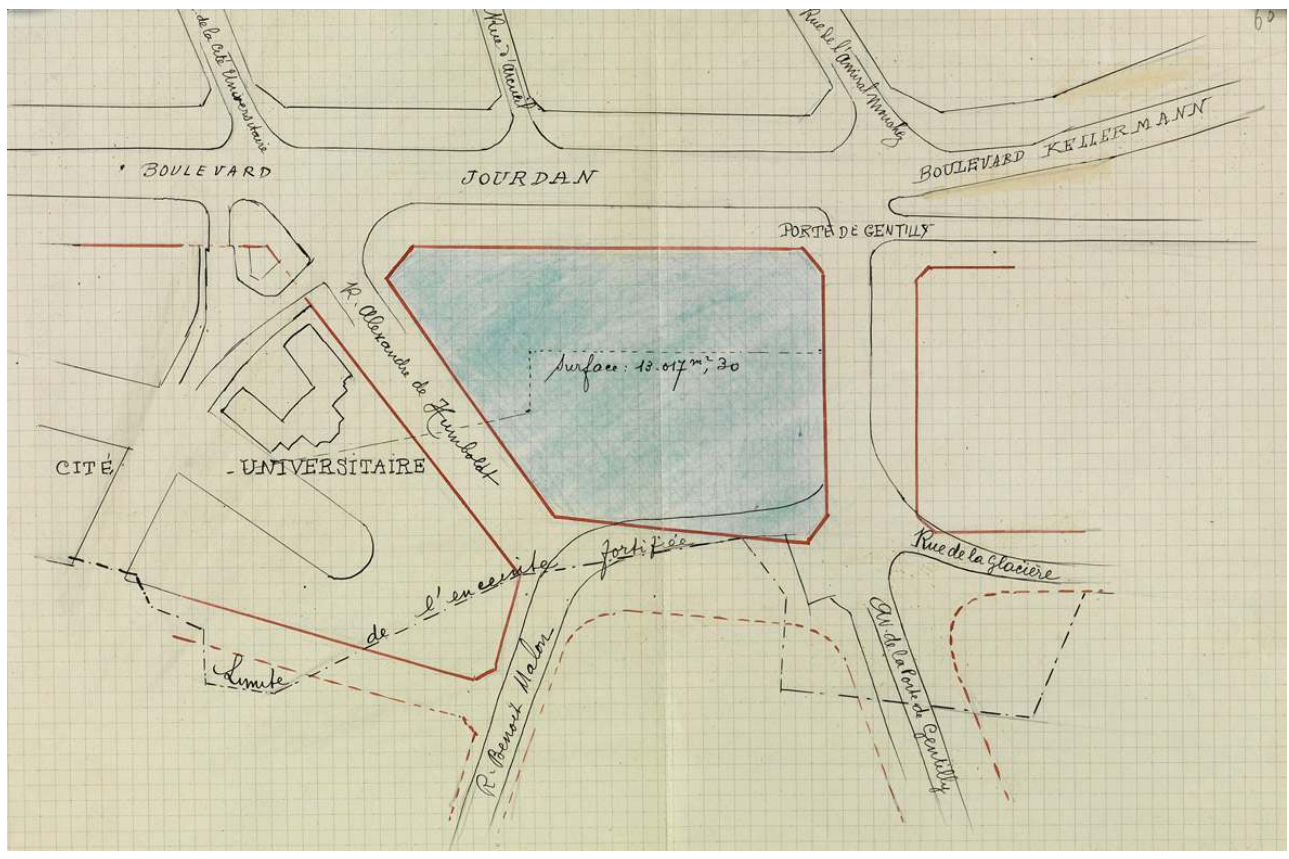

Plan de l'« îlot 19 du XIVe arrondissement » ou « terrain David-Weill », 1927 ; en pointillé, la limite de l'enceinte fortifiée séparant l'îlot des terrains zoniers que la Fondation nationale souhaite incorporer au domaine de la Cité. AN, AJ16 /7032. Repro. Ayrault, Philippe.

(c) Conseil régional d'île-de-France, service Patrimoines et Inventaire, 2009. [Base Mémoire: 20097501777NUC4A] 


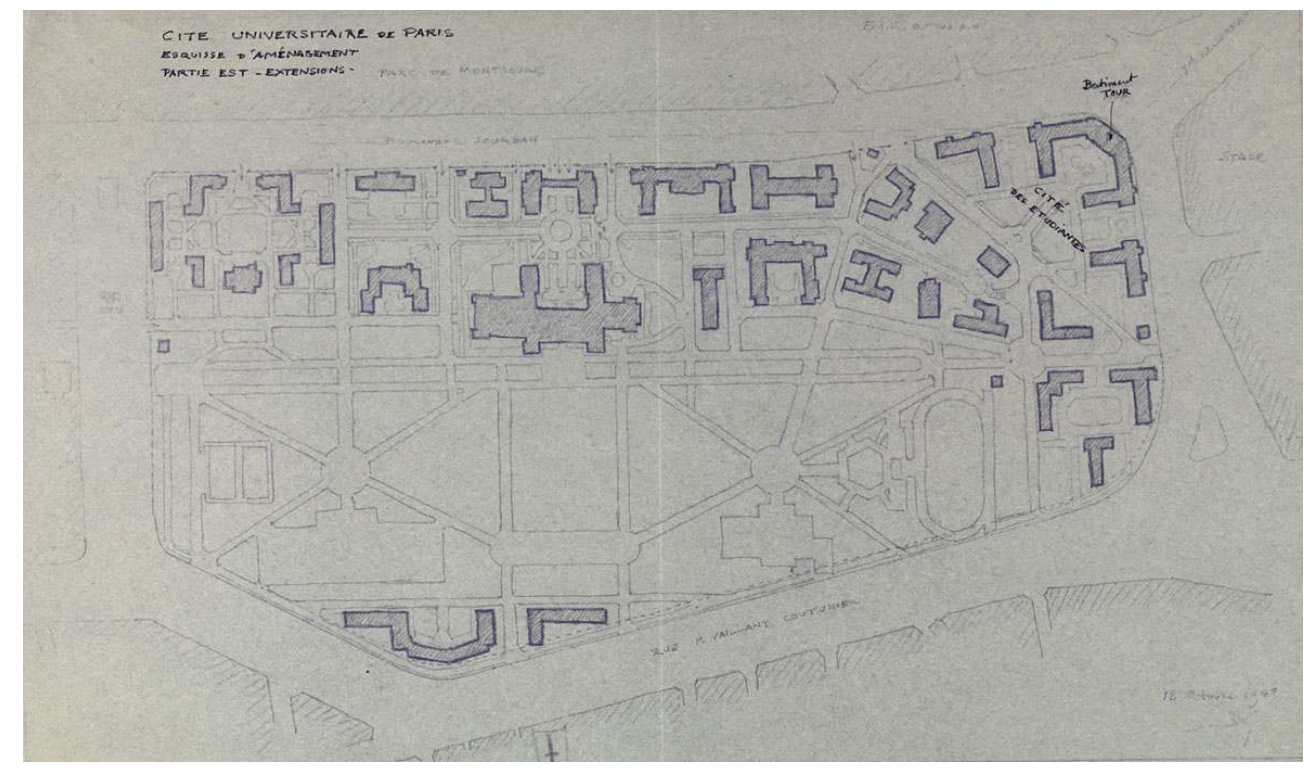

Projet de «Cité des étudiantes » à l'extrême est du domaine, entre la rue Alexandre-de-Humboldt et l'avenue de la Porte de Gentilly, vers 1934. IFA, 47/40. Repro. Bazaud, Chloé.

(c) Conseil régional d'île-de-France, service Patrimoines et Inventaire, 2009. [Base Mémoire : 20097500286NUC4A]

\section{Sur le boulevard Jourdan, en face de la Cité universitaire : le terrain de remonte}

7 Même agrandi des $13000 \mathrm{~m}^{2}$ de l'îlot David-Weill, le domaine risque de se trouver rapidement à l'étroit, compte tenu du développement pris en si peu de temps par la Cité. Reprenant une proposition déjà présentée en 1920, la Fondation nationale demande alors l'affectation à l'Université des terrains de l'école de dressage (ou dépôt de remonte) du boulevard Jourdan, que l'autorité militaire doit remettre à l'administration des Domaines ${ }^{9}$

Ce terrain, acquis par l'État en 1841 dans la perspective de protéger la capitale, a la forme d'un trapèze irrégulier d'une superficie de 4,63 ha, entre le boulevard au sud, la rue de la Tombe Issoire à l'ouest et l'avenue Reille au nord ${ }^{10}$; traversé en diagonale par les canalisations qui conduisent les eaux de la Vanne et du Loing du réservoir de Gentilly à ceux de Montsouris, il est occupé par diverses constructions : écuries et manège à l'est zone plantée de 125 gros arbres et de petits arbres fruitiers -, autres écuries et lavoir dans sa partie ouest, dont une parcelle de $19000 \mathrm{~m}^{2}$ a été, le 5 avril 1924, concédée au ministère du Travail et de l'Hygiène qui y a fait édifier les baraquements du Centre d'hébergement des réfugiés et sans-abri du département de la Seine (fig. $\left.\mathbf{n}^{\circ} \mathbf{8}\right)$. Le $1^{\text {er }}$ mai 1929, la commission interministérielle chargée d'étudier la demande de la Fondation, lui donne un accord unanime, sous réserve toutefois de la mise à la disposition de l'administration des Beaux-Arts d'un terrain affecté à la reconstruction des ateliers d'artistes du quai de l'Alma dits du «dépôt des Marbres». Elle propose de ramener à 10 millions le prix des 4 ha que l'administration des Domaines a évalué à plus de 14. Par décret du 27 avril $1930^{11}$, celle-ci affecte à la Fondation la partie de l'ancien dépôt de remonte non occupée par le centre d'hébergement, d'une superficie approximative de 
2,22 ha, moins une large bande de $26 \mathrm{~m}$ 79, en bordure du boulevard Jourdan, qui doit être incorporée ultérieurement à la voirie. Prévu à Bobigny dès janvier 1929, puis sur un terrain dépendant de l'ancien bastion 94, à l'angle du boulevard Masséna et du quai d'Ivry le 30 décembre 1930, le transfert du centre d'hébergement, qui abrite encore 1500 personnes, n'est plus envisagé par le Conseil général qu'à l'horizon 1934 ou 1935, malgré les doléances de la Fondation contre « un voisinage regrettable à tous égards pour l'œuvre de la Cité universitaire $»^{12}$.

Figure 8

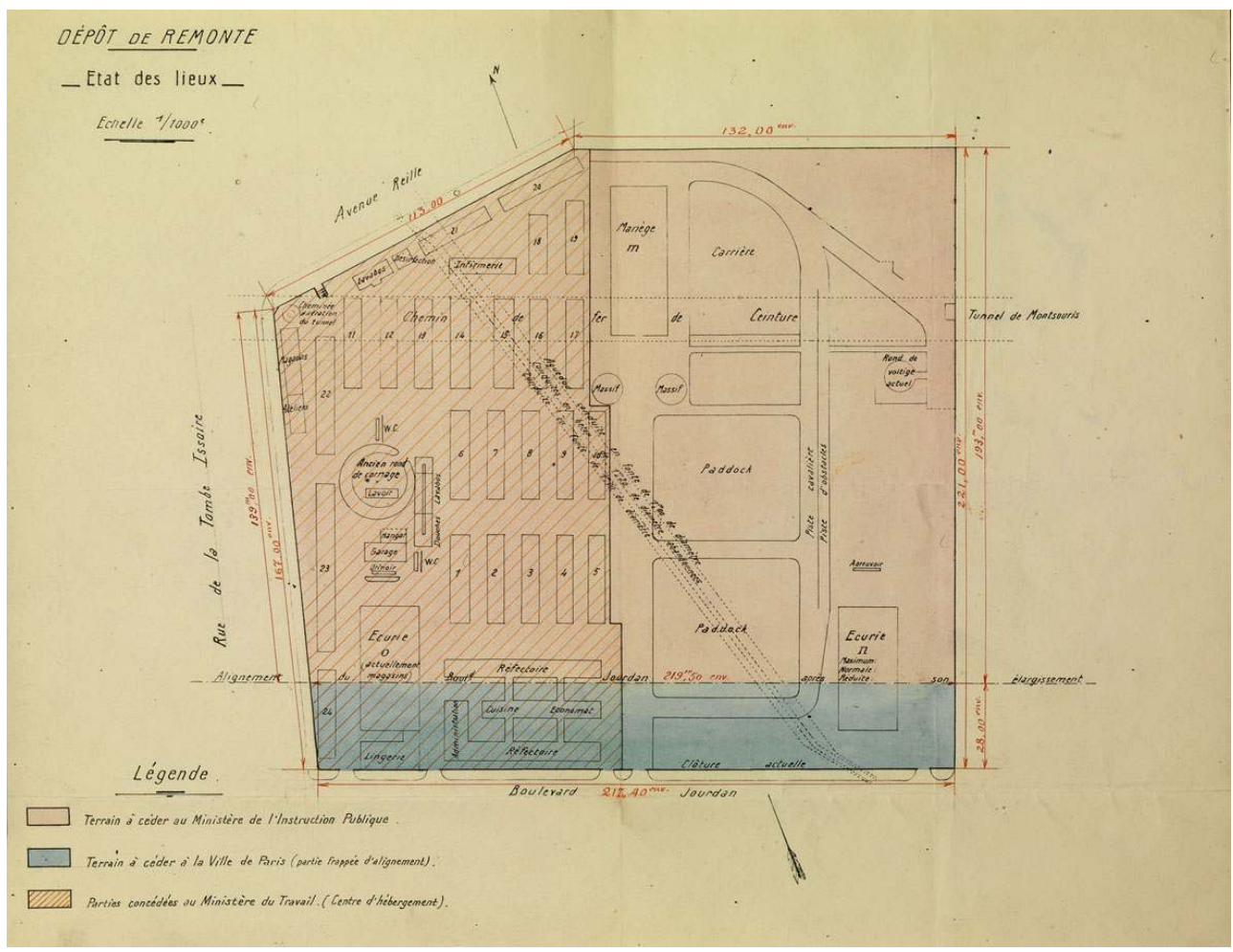

Plan du dépôt de remonte, avec, d'un côté, les baraquements du centre d'hébergement et, de l'autre, les paddocks, écuries et manège. En bleu, la parcelle frappée d'alignement; vers 1930. AN, AJ16 17027. Repro. Ayrault, Philippe.

(c) Conseil régional d'île-de-France, service Patrimoines et Inventaire, 2009. [Base Mémoire : 20097501740NUC4A]

\section{4 ha sur la commune de Gentilly}

Tout en poursuivant l'acquisition des 4 ha de l'école de dressage, le comité de direction de la Fondation nationale, qui craint une rapide saturation des terrains, mise sur un projet d'extension à l'autre extrémité du domaine, du côté de la commune de Gentilly. Les terrains situés au-delà de la porte d'Arcueil et de la rue de la Tombe Issoire étant déjà affectés, par la Ville de Paris, à d'autres usages, la Cité est dans l'impossibilité de s'étendre en largeur et ne peut trouver de terrains disponibles qu'en bordure de sa limite sud, audelà du futur parc universitaire. Les services de la Ville ont étudié la question et évalué à 10 millions de $\mathrm{F}$ la superficie de 4 ha constituée par une sorte de triangle isocèle compris entre la zone et la rue de Montrouge ${ }^{13}$ (fig. $\mathbf{n}^{\circ} 9$ ). 


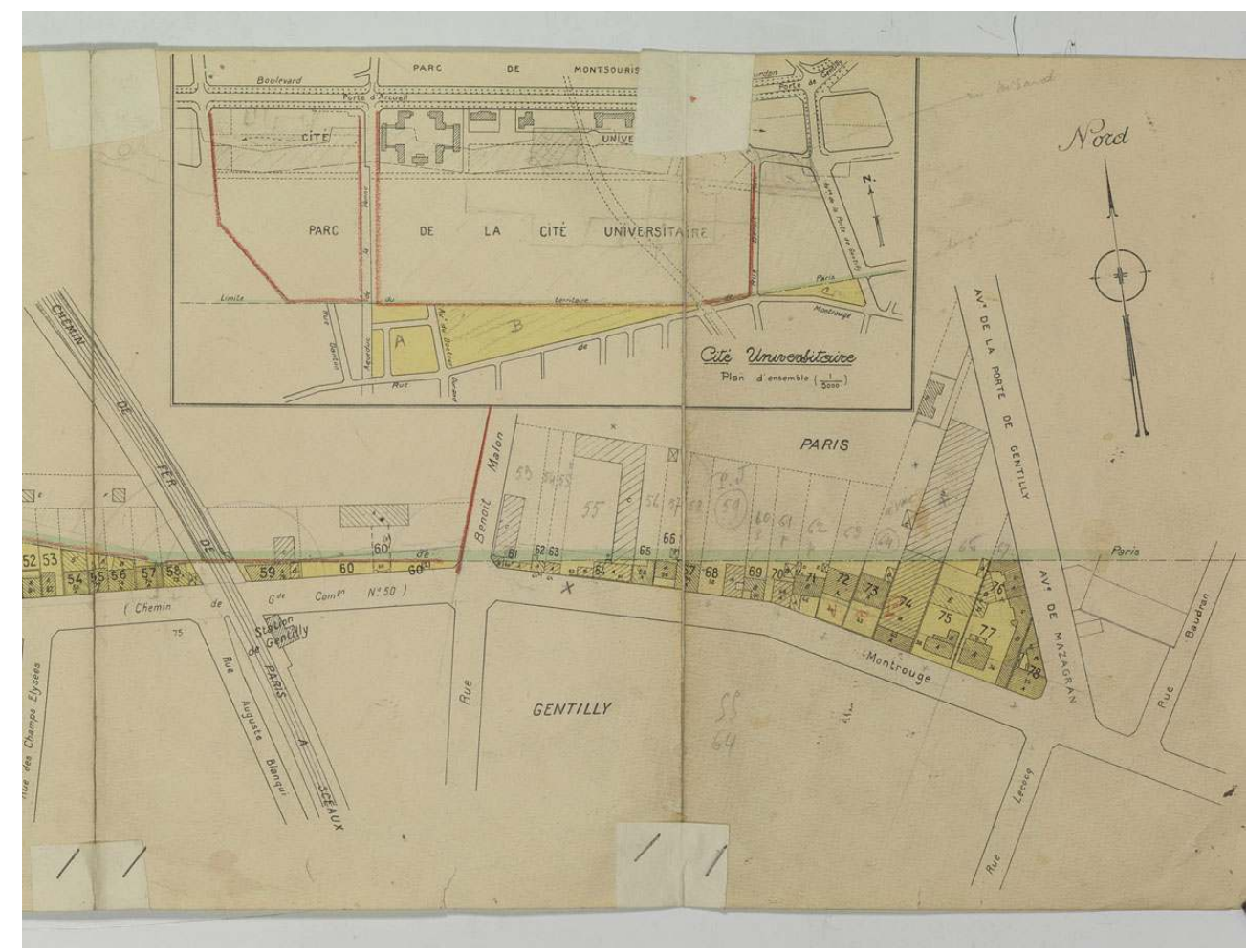

Terrains de Gentilly expropriés en 1928 : plan d'ensemble, en haut, dans l'encadré ; en bas, détail de la partie est. CAC, 20090013/243. Repro. Kruszyk, Laurent.

(c) Conseil régional d'île-de-France, service Patrimoines et Inventaire, 2009. [Base Mémoire : 20097500886NUC4A]

10 L'expropriation de ces terrains, en vue de l'agrandissement du domaine de la Cité « sur le territoire de Gentilly et en bordure du parc de la zone ", est prononcée par la loi du 10 juillet 1928. Une clause maintient les droits de la Ville de Paris sur la parcelle occupée par ses réservoirs entre l'aqueduc de la Vanne et la rue du Docteur-Durand, sous réserve d'une rétrocession à l'État pour les besoins de la Cité universitaire, «au cas où elle jugerait utile de les déplacer $»$.

Un délai de près de cinq ans s'écoule entre la promulgation de la loi et la prise de possession des terrains : le jury chargé de fixer les indemnités dues aux 110 propriétaires et 150 locataires (qui occupent 78 lots d'expropriation) tient sa première séance en juin 1930 seulement $^{14}$. La lenteur de la procédure a pour conséquence de majorer considérablement les frais de l'opération, dont le coût final s'élève à environ 36 millions ${ }^{15}$, soit plus du double de ce qui aurait été nécessaire si les travaux du jury avaient débuté immédiatement après le vote de la loi. Celle-ci a prévu que l'opération donnerait lieu à une réduction correspondante de la subvention versée à la Cité universitaire : en 1927 en effet, le Parlement a décidé d'affecter, à partir de 1928, une somme de 18 millions de $\mathrm{F}$ au développement de la Cité, somme à verser en 4 annuités, l'une de 3 millions et les 3 autres de 5 millions chacune. En 1932, pour solder les opérations d'expropriation, la Fondation nationale renonce ainsi à toucher une subvention de $3700000 \mathrm{~F}^{16}$ et en 1933 abandonne de même un surplus indispensable de $1038149 \mathrm{~F}$. À la fin de l'année, les anciens propriétaires ont tous quitté les immeubles expropriés - à l'exception d'un seul qui «a tenu à affirmer son droit de rester en possession jusqu'au 23 avril prochain et, bien qu'il 
ait déjà évacué l'immeuble, s'est refusé jusqu'ici à remettre les clés ${ }^{17}$ » - : en 1934 la Cité occupe désormais l'ensemble du territoire qui lui a été affecté par arrêté du 8 juin 1932 (fig. $\left.n^{\circ} 10\right)$.

Figure 10

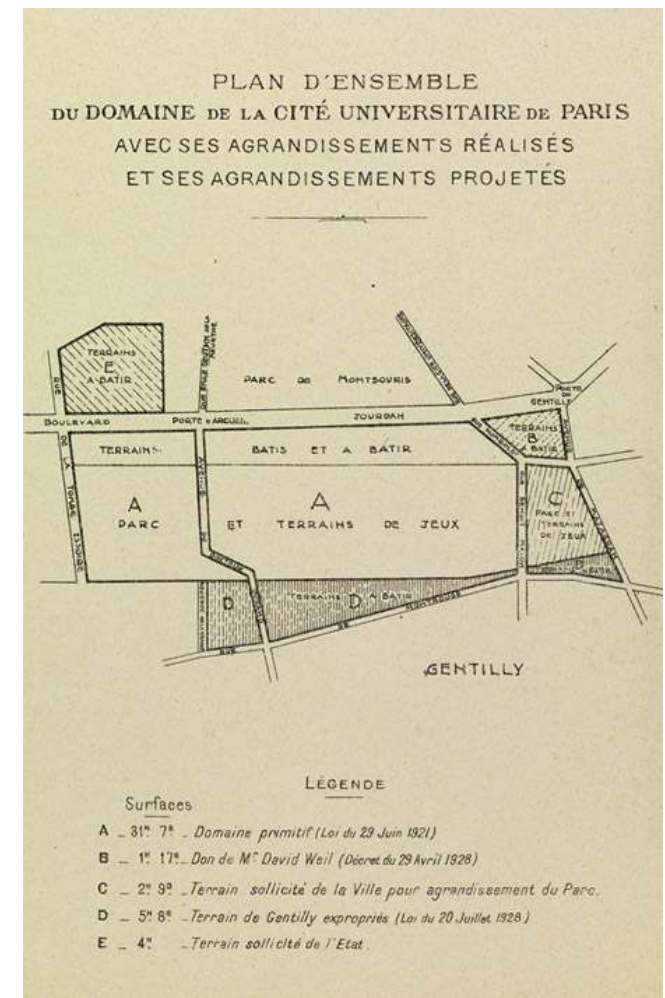

Plan d'ensemble de la Cité universitaire avec ses agrandissements successifs, 1929 ; en 1930, les terrains du dépôt de remonte (E) seront intégrés au domaine, qui couvrira alors un peu plus de 42 ha ; seule la parcelle C restera en attente d'annexion. AN, AJ16 /7034. Repro. Ayrault, Philippe.

(c) Conseil régional d'île-de-France, service Patrimoines et Inventaire, 2009. [Base Mémoire: 20097501789NUC4A]

\section{La suppression de la voie d'isolement et son report sur la rue de Montrouge, l'annexion au territoire de Paris}

L'incorporation des terrains de Gentilly au domaine de la Cité universitaire pose toutefois un certain nombre de problèmes qui entraînent la modification des projets primitifs d'aménagement.

Le parc de la Cité devait, à la limite de Paris, être séparé du territoire de la commune de Gentilly par une voie d'isolement, suivant le principe d'aménagement auquel la Ville de Paris se conforme sur tout le pourtour de la zone. Mais cette voie devient désormais sans objet : son maintien aurait pour conséquence de scinder en deux le domaine de la Cité ; pour éviter cet inconvénient et réunir les terrains en un seul bloc, il convient qu'elle soit reportée à la limite des terrains acquis par l'État pour l'extension de la Cité, en suivant le tracé de la rue de Montrouge élargie à $20 \mathrm{~m}$. L'exécution de ce projet rend indispensable un échange de terrains entre l'État et la Ville de Paris : celle-ci incorporant au parc de la Cité - qui gagne ainsi un hectare - les terrains sur lesquels elle devait ouvrir une voie le 
long de la zone, l'État doit lui céder gratuitement, en compensation, les terrains permettant de transformer la rue de Montrouge en boulevard.

Un intérêt tout aussi puissant, pour la Cité, s'attache à ce que la cession des terrains de Gentilly s'accompagne de leur annexion au territoire de Paris. La plus importante partie de son domaine étant située sur ce territoire, il serait illogique que l'autre partie continue à dépendre de la commune de Gentilly, et que de ce fait, la Ville de Paris ait à réaliser une opération d'urbanisme sur le sol d'une autre commune. Faire dépendre la Cité de deux administrations municipales aboutirait à en compliquer la gestion, ce que le comité de direction fait valoir au préfet de la Seine dans une lettre du 3 janvier $1935^{18}$. L'enquête règlementaire instruite dans les mois qui suivent auprès des six habitants domiciliés sur ces terrains ne recueille que des avis favorables ; les déclarations des conseils municipaux de Gentilly et de Paris, dans leurs séances respectives des 4 juin et 31 décembre 1936, le sont tout autant ${ }^{19}$. Le premier se borne à demander en compensation l'élargissement immédiat à $20 \mathrm{~m}$ de la rue de Montrouge, par la Ville de Paris et à ses frais, ainsi que la construction par la Cité universitaire de bâtiments en bordure de cette voie, «ce qui aurait pour effet de donner de l'animation au commerce de ce quartier ».

Figure 11

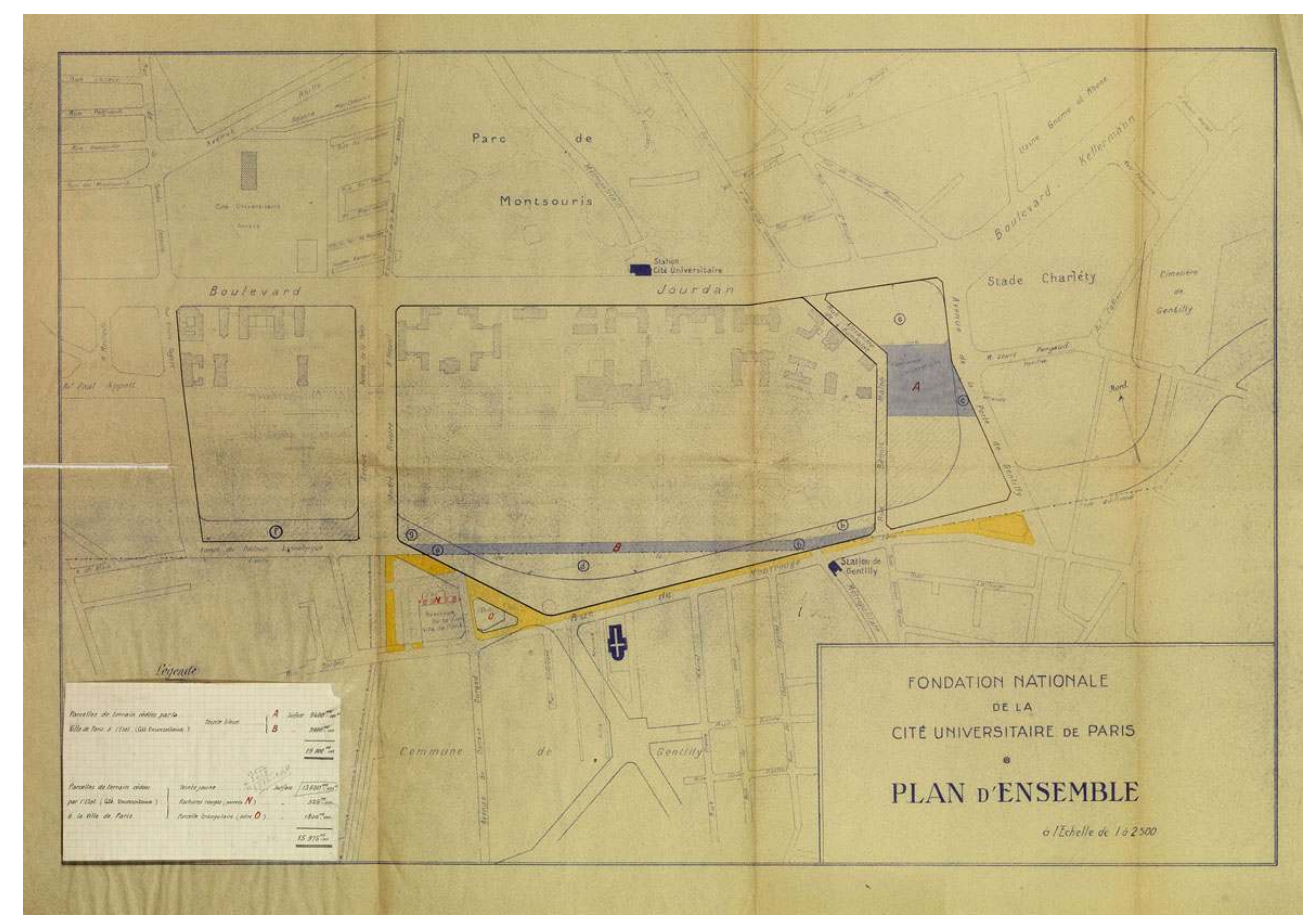

Suppression de la voie d'isolement, reportée sur la rue de Montrouge : l'échange de terrains mis au point en 1936, sanctionné par décret du 13 septembre 1941 : en bleu, les parcelles cédées par la Ville de Paris à l'État, en jaune, celles que l'État abandonne à la Ville de Paris, augmentées des deux petites parcelles N (hachures rouges) et O en forme de triangle. CAC, 20090013/243. Repro. Kruszyk, Laurent.

(c) Conseil régional d'île-de-France, service Patrimoines et Inventaire, 2009. [Base Mémoire : 20097500875NUC4A]

Simultanément, la préfecture de la Seine procède à la mise au point de l'échange de terrains consécutif au projet d'aménagement des voies publiques aux abords de la Cité ${ }^{20}$ (fig. $\mathbf{n}^{\circ} 11$ ). Celui-ci, qui porte sur une surface évaluée à $23525 \mathrm{~m}^{2}$, impliquerait pour l'État la cession de $13650 \mathrm{~m}^{2}$ destinés à l'ouverture et l'élargissement de plusieurs voies ${ }^{21}$, 
ainsi que de trois parcelles situées à proximité des rues de Montrouge et de Mazagran ${ }^{22}$, soit au total une surface de $15975 \mathrm{~m}^{2}$. De son côté, la Ville de Paris abandonnerait, outre l'assiette de la voie d'isolement de la zone $\left(9600 \mathrm{~m}^{2}\right)$, un «terrain de $9400 \mathrm{~m}^{2}$ destiné à servir de jardin aux jeunes filles et dépendant pour partie de l'ancienne fortification $\left(1260 \mathrm{~m}^{2}\right)$ et pour partie de la zone $\left(8140 \mathrm{~m}^{2}\right) »$ : il s'agit du terrain situé entre la rue Benoît-Malon et l'avenue de la Porte de Gentilly, dans le prolongement de l'îlot DavidWeill, que la Ville de Paris s'était, jusque-là, toujours refusée à céder à la Cité universitaire. Celle-ci s'agrandirait ainsi d'une superficie totale de $19000 \mathrm{~m}^{2}$, dont la plus grande partie $\left(17740 \mathrm{~m}^{2}\right)$, située sur la zone, reste grevée de la servitude non aedificandi. Minime du point de vue des surfaces ( $3000 \mathrm{~m}^{2}$ environ au bénéfice de l'État), la différence entre les deux catégories de terrain est importante pour celui des valeurs : les terrains de la zone cédés par la Ville sont estimés à 2,7 millions de F, contre 11,3 millions pour les terrains hors zone remis par l'État. Le 5 novembre 1936, le ministère des Finances donne cependant son aval à un échange sans soulte, compte tenu des « charges supplémentaires assumées par la Ville de Paris pour l'aménagement et l'entretien des voies publiques prévues ", à l'occasion d'une opération d'urbanisme conçue dans l'intérêt exclusif de la Cité universitaire ${ }^{23}$. Dans sa séance du 31 décembre suivant le conseil municipal ratifie les modalités de l'accord. La cession réciproque peut avoir lieu sans recourir à la procédure d'échange - qui nécessiterait le vote d'une loi - sous réserve de la déclaration d'utilité publique des travaux à entreprendre. «Étant donné l'urgence de ces travaux et les demandes pressantes de M. Honnorat », le ministre des Finances consent, le 7 juin 1937, «à une prise de possession immédiate des terrains à céder de part et d'autre " ${ }^{24}$, avant même la déclaration d'utilité publique qui intervient le 26 novembre suivant; en 1938, la Ville de Paris peut ainsi ouvrir " une voie publique entre l'avenue André-Rivoire et le carrefour de l'avenue Paul-Vaillant-Couturier et la rue Dedouvre à Gentilly ». Mais les échanges ne deviennent définitifs qu'après avoir été sanctionnés par le décret du 13 septembre 1941 fixant les nouvelles limites de Paris avec la commune de Gentilly.

\section{Après la seconde guerre mondiale : la création du boulevard périphérique et les atteintes au domaine de la Cité universitaire}

16 Au lendemain de la seconde guerre mondiale, le domaine de la Cité universitaire, porté à plus de 40 ha par trois agrandissements successifs, a atteint son développement maximal. 


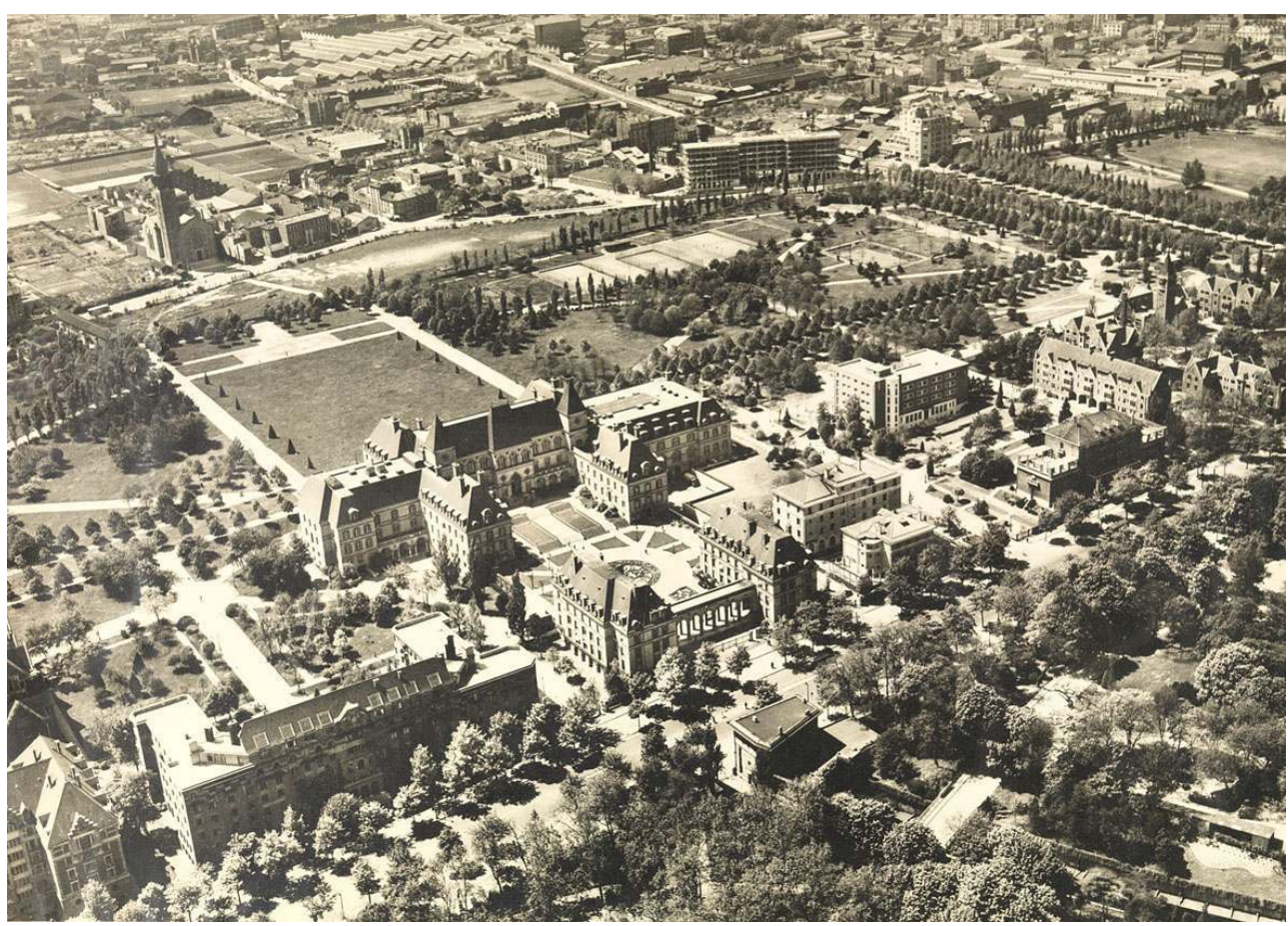

Vue aérienne de la Cité universitaire vers 1950 (partie centrale) : les « terrains de Gentilly » sont encore vacants ; au-delà de la rue de Montrouge, la Maison des Arts et Métiers (inaugurée en novembre 1950) est en cours de construction. AN, AJ16/7034. Repro. Ayrault, Philippe.

(c) Conseil régional d'Île-de-France, service Patrimoines et Inventaire, 2009. [Base Mémoire: 20097500315NUC4A]

Après un temps d'arrêt de douze années dû aux menaces de guerre, à la guerre ellemême, puis aux difficultés financières et économiques qui l'ont suivie, la Cité reprend un nouvel essor sous l'impulsion de son nouveau directeur Raoul Dautry, ancien ministre du MRU. Dès 1951, elle compte 21 fondations dans un parc pourvu de nombreuses installations sportives (fig. $\mathbf{n}^{\circ} \mathbf{1 2}$ ) et les implantations prévues risquent de conduire, faute de place, à "un resserrement fâcheux des maisons ». La situation est d'autant plus critique que le plan d'aménagement de Paris et de la banlieue parisienne prévoit la réalisation d'une opération routière de grande envergure ayant pour effet de réduire sérieusement la surface des terrains à bâtir dans la zone encore disponible. Envisagé dès avant la guerre, l'élargissement à $50 \mathrm{~m}$ de la rue de Montrouge - désormais avenue PaulVaillant-Couturier, tronçon de «la voie circulaire ou périphérique qui doit être ouvert tout le long du territoire de Paris » - conduit à prélever une surface de 2,90 ha ${ }^{25}$ sur la lisière sud du domaine universitaire, amputation qui ouvre à la Fondation nationale des droits à l'obtention de terrains de compensation. Des accords verbaux passés en 1943 ont proposé la réalisation de divers échanges ${ }^{26}$ qui servent de base à l'élaboration d'un nouveau projet (décembre 1949 ${ }^{27}$ ), dont l'exécution se trouve elle aussi différée pour permettre l'étude du "débouché éventuel sur cette rocade de l'autostrade Paris-Orly $»^{28}$. Les modalités définitives de l'échange (fig. $\mathbf{n}^{\circ}{ }^{13}$ ) sont établies en $1952^{29}$ : l'État cède à la Ville de Paris une surface totale de $19510 \mathrm{~m}^{2}$, constitués pour l'essentiel $\left(14810 \mathrm{~m}^{2}\right)$ de terrains provenant des expropriations menées à Gentilly - donc constructibles - et pour un peu plus du quart d'anciens terrains zoniers. En contrepartie, la Ville abandonne le terrain d'assiette de la rue Alexandre-de-Humboldt ${ }^{30}$, soit $2780 \mathrm{~m}^{2}$, ainsi que trois petites 
parcelles situées près du réservoir de la Vanne d'un total inférieur à $1000 \mathrm{~m}^{2}$. Pour les terrains loués, l'État reçoit un peu plus $\left(14300 \mathrm{~m}^{2}\right)$ que la Ville ne lui reprend $\left(10420 \mathrm{~m}^{2}\right)$; parmi ces nouveaux terrains loués à la Cité universitaire, figurent un lot de plus d'un hectare situé au sud de l' «îlot 19 » (terrain David-Weill) agrandi en 1941, ainsi que le terrain d'assiette de la rue Benoît-Malon $\left(3150 \mathrm{~m}^{2}\right)$, une fois son déclassement prononcé : grâce à ces remaniements, le domaine gagne en homogénéité (par la suppression de deux voies publiques qui le traversent) et s'étend à l'est jusqu'à l'avenue de la Porte de Gentilly, à partir de laquelle il forme un quadrilatère d'un seul tenant jusqu'à l'avenue AndréRivoire à l'ouest (fig. $\left.\mathbf{n}^{\circ} \mathbf{1 4}\right)$.

Figure 13

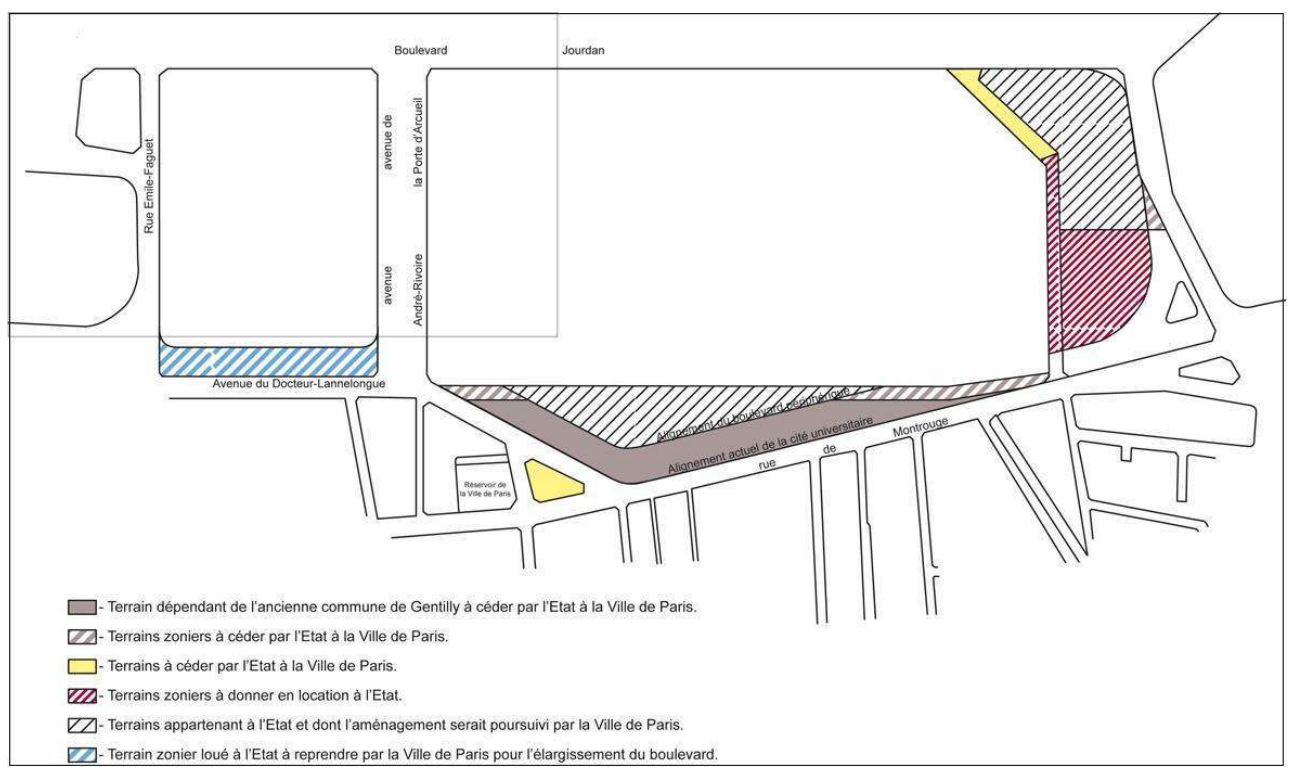

Reconstitution du plan joint au « procès-verbal de remise provisoire des terrains échangés entre la Ville de Paris et l'État», du 23 septembre 1952. Carto. Bétored, Diane.

(c) Conseil régional d'île-de-France, service Patrimoines et Inventaire, 2010. 


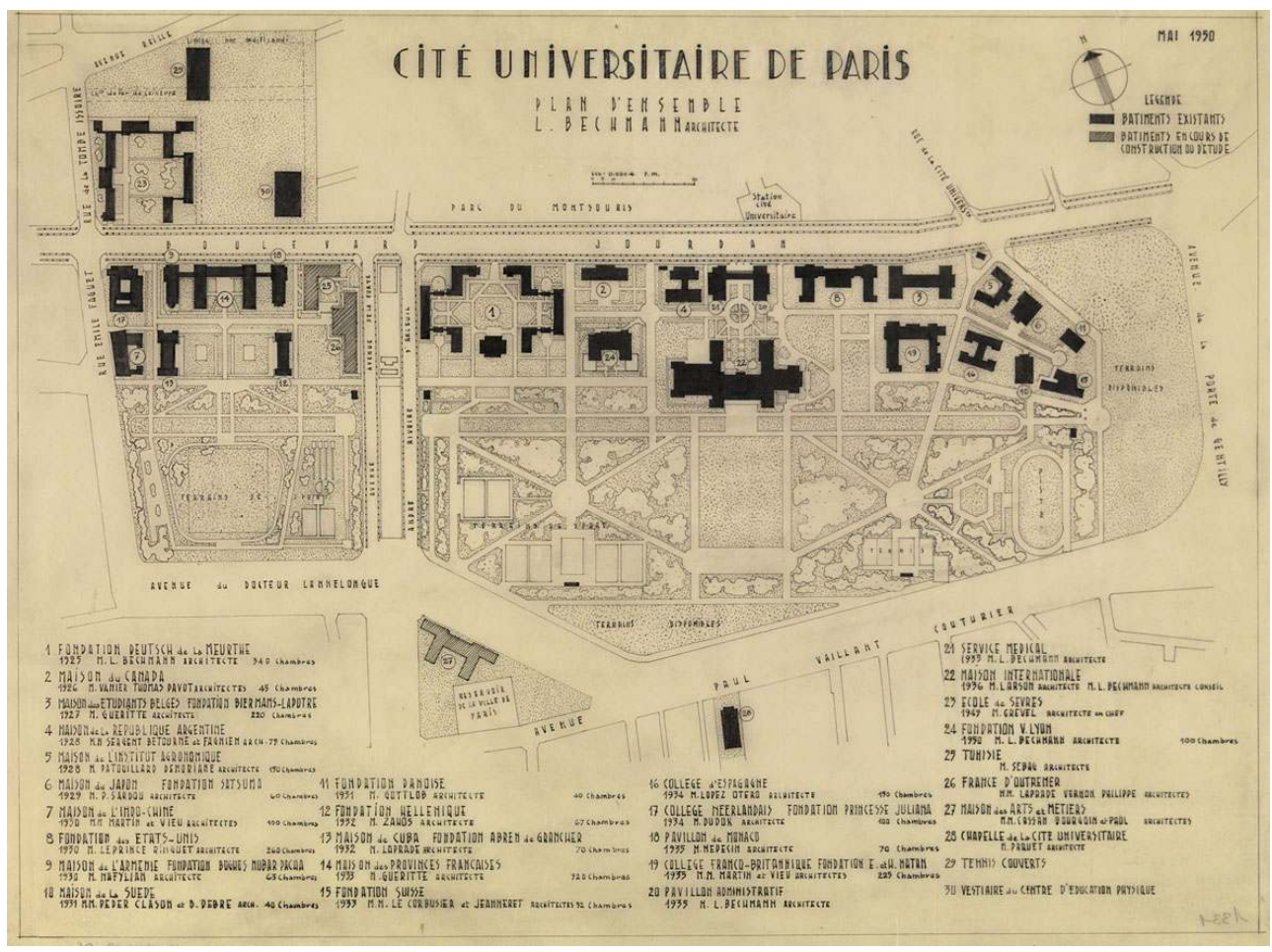

Plan d'ensemble, mai 1950 : I'« îlot 19 » et les terrains zoniers adjacents ont été refondus en une seule parcelle ; le nouveau tracé de la rue Paul-Vaillant-Couturier (futur boulevard périphérique) a empiété sur les « terrains de Gentilly », encore disponibles. IFA, 47/40. Repro. Bazaud, Chloé.

(c) Conseil régional d'Île-de-France, service Patrimoines et Inventaire, 2009. [Base Mémoire : 20097500282NUC4A]

18 La remise provisoire des terrains échangés donne lieu à l'établissement d'un procèsverbal le 23 septembre 1952, qui subordonne la remise définitive à un accord sur les modalités financières de l'opération: une soulte doit être versée par l'administration municipale à l'État, en raison de la disproportion de l'échange - $15785 \mathrm{~m}^{2}$ au profit de la Ville -, déséquilibre qu'un nouvel élargissement du boulevard périphérique accentue six ans plus tard (fig. $\mathbf{n}^{\circ} \mathbf{1 5}$ ); le raccordement de l'autoroute du sud nécessite en effet le prélèvement d'une bande de terrain supplémentaire de $10 \mathrm{~m}$ sur toute la longueur de l'emprise sud du domaine ${ }^{31}$. 


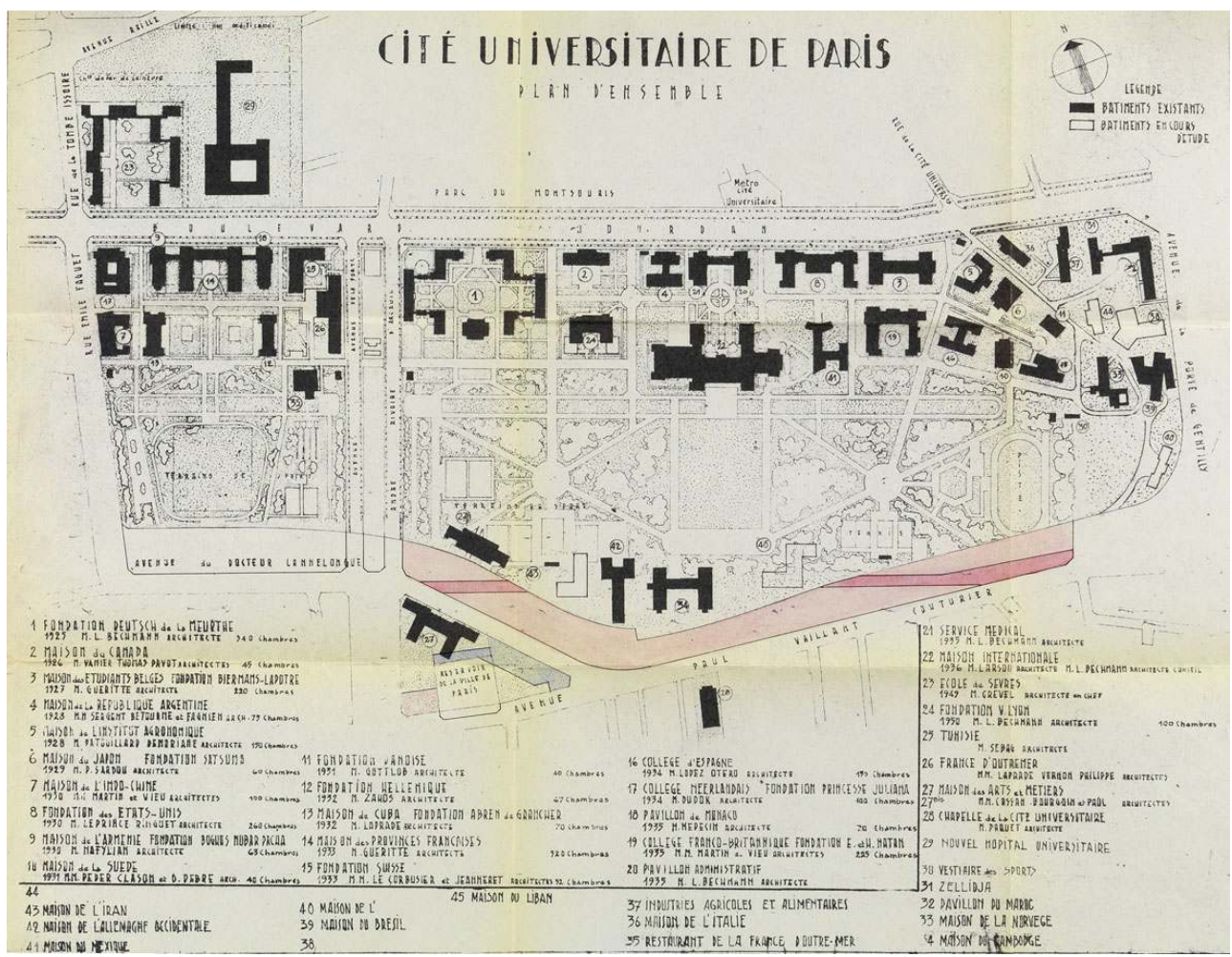

Plan d'ensemble, vers 1960 ; en rose, le tracé du futur boulevard périphérique ; des bâtiments, réalisés ou en projet, occupent l'îlot est et les anciens «terrains de Gentilly » au sud. AN, AJ16 /7027. Repro. Ayrault, Philippe.

(c) Conseil régional d'île-de-France, service Patrimoines et Inventaire, 2009. [Base Mémoire : 20097501738NUC4A]

Malgré le «tort sérieux porté à la Cité par la réalisation de ce projet » qui nuit aux « engagements pris à l'égard de plusieurs donateurs »- les maisons du Cambodge et de l'Allemagne ne seront plus « isolées d'une voie de circulation très intense » - la Fondation nationale donne son accord de principe par lettre du 17 octobre 1956, deux ans avant la prise de possession effective des terrains par les services de la préfecture ${ }^{32}$ (fig. $\mathbf{n}^{\mathbf{0}} \mathbf{1 6}$ ). Elle juge dès lors utile de régulariser cette nouvelle cession par la rectification du procèsverbal d'échange du 23 septembre 1952, mais le préfet estime cette demande "prématurée " ${ }^{33}$. Une tentative de règlement a lieu lors de la conférence du 19 février 1959 : la Fondation demande, en échange des terrains perdus, la remise du « maximum de surface sur les parcelles appartenant à la Ville de Paris (service des eaux) à l'est de la maison des Arts et Métiers» et le versement d'une indemnité, fixée plus tard à $1960000 \mathrm{~F}$, sur la base de $120 \mathrm{~F}$ par $\mathrm{m}^{2}$. «Malgré les accords réitérés donnés par [ses] lettres du 19 mai et du 25 août 1960 » et de nombreuses démarches, la Fondation est encore « sans nouvelle de cette affaire » en $1962^{34}$. 


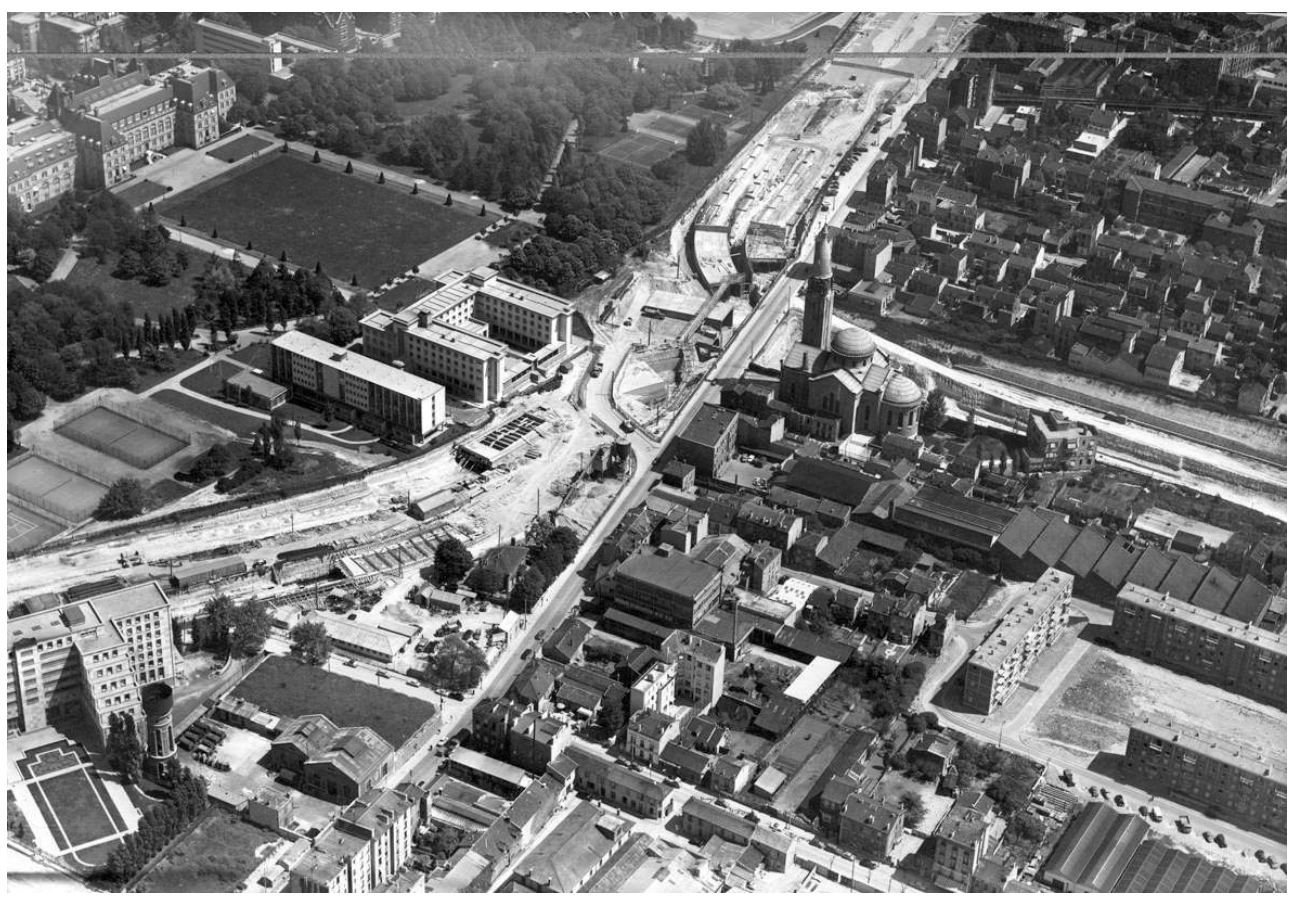

Le périphérique en cours de construction. Phot. IGN/Compagnie aérienne française.

(c) Archives photographiques, CIUP.

\section{Les terrains du Chaperon Vert}

20 Afin de réparer l'amputation prévisible de terrains à bâtir que le projet de création du boulevard périphérique lui fera subir, la Fondation nationale s'est portée candidate, dès 1948, à l'acquisition de terrains nus existant au sud et à proximité immédiate des emprises de la Cité. Le Parlement vient alors d'abroger l'ordonnance du 24 octobre 1945 qui avait réservé une soixantaine d'hectares sur les territoires d'Arcueil et de Gentilly à la création d'un centre universitaire et scientifique pour la région parisienne ${ }^{35}$. En août 1948, la Ville de Paris est autorisée à acquérir, pour le compte de la Cité, un îlot de 16,80 ha dit du Chaperon Vert délimité par l'avenue Paul-Vaillant-Couturier, l'avenue du Docteur-Durand, la rue Jean-Jaurès et l'avenue Ernest-Renan, mais sur ce total, les mairies de Gentilly et d'Arcueil ont décidé, par délibérations des 12 et 20 mai 1948, de réserver deux parcelles d'environ 4 ha chacune en vue de la réalisation de cités jardins et de groupes d'HBM. Un partage équitable des terrains est alors étudié par la préfecture de la Seine, mais aucun accord ne peut être conclu: le 13 avril 1949, le conseil municipal de Gentilly confirme son opposition à la vente de terrains qui «constituent la ressource essentielle de la commune pour réaliser des constructions nouvelles ». Le différend est tranché par le Comité d'aménagement de la région parisienne dans sa séance du 2 juillet 1951: les terrains situés à l'est de la rue Joseph-Staline (ex-rue du Docteur-Durand) reviendront à l'office intercommunal, tandis que les espaces libres situés de l'autre côté seront attribués à la Cité universitaire, à laquelle conduira le large boulevard planté prévu sur l'emplacement de la dérivation des eaux de la Vanne (fig. $\mathbf{n}^{\circ} \mathbf{1 7}$ ). 


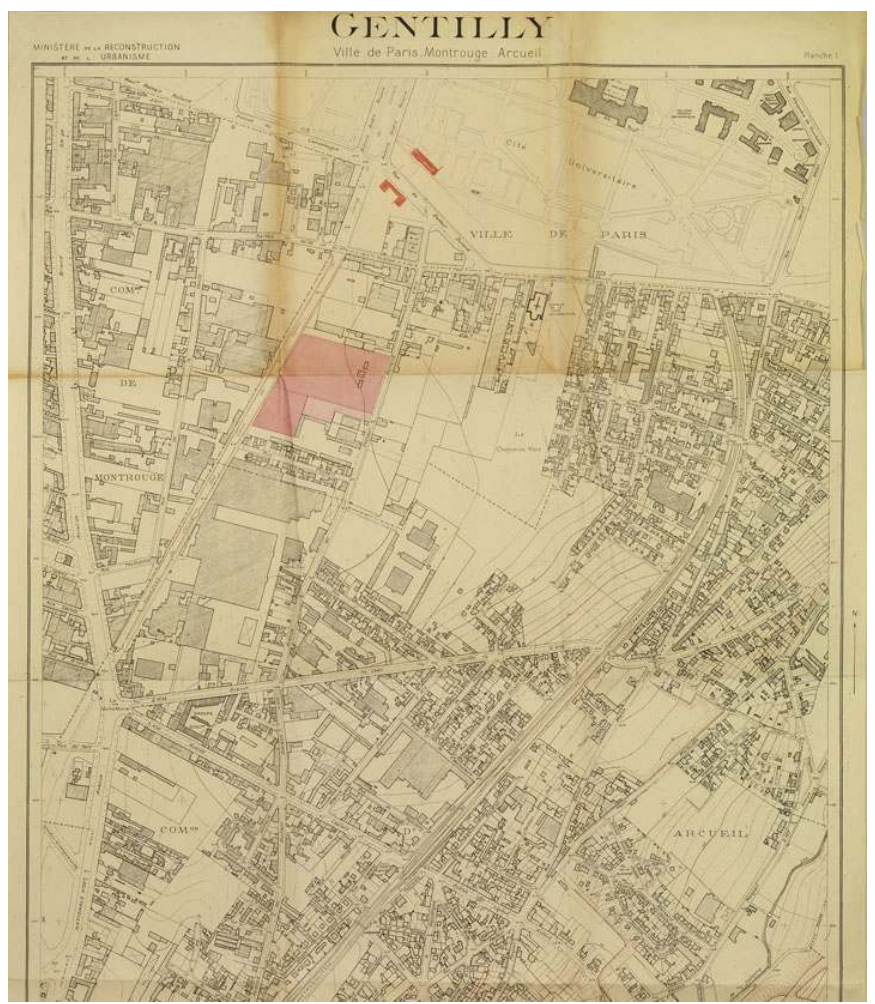

Plan du terrain du Chaperon Vert affecté à l'agrandissement de la Cité universitaire sur le territoire de la commune de Gentilly. CAC, 20090013/244. Repro. Kruszyk, Laurent.

(c) Conseil régional d'île-de-France, service Patrimoines et Inventaire, 2009. [Base Mémoire : 20097500884NUC4A]

Le comité de direction et le maire de Gentilly acceptent cette solution de compromis qui permet de concilier les intérêts en présence. Le premier se propose alors d'affecter le nouveau terrain (3,57 ha) à la construction d'un hôpital universitaire destiné à remplacer le pavillon médical dont «les aménagements ne correspondent plus aux besoins qui se manifestent, singulièrement depuis l'extension des avantages de la sécurité sociale aux étudiants ${ }^{36}$. Mais en 1954, préférant implanter l'hôpital sur les terrains sis au 44, boulevard Jourdan (ancien dépôt de remonte), occupés jusque-là par des équipements sportifs (fig. $\mathbf{n}^{\circ} \mathbf{1 8}$ ), il réserve le terrain du Chaperon Vert au transfert de ces installations qui couvrent une superficie d'environ 2 ha. Par délibération du 16 décembre 1954, la commune de Gentilly remet alors en cause l'affectation du terrain, sous prétexte de l'abandon du projet d'hôpital - tandis qu'en octobre 1955, la « commission instituée pour favoriser une meilleure répartition des industries sur l'ensemble du territoire » en affecte une partie à la société Les transformateurs de mesure E. Walter, qui souhaite construire de nouveaux ateliers au nord de son usine située 64 , avenue Staline ${ }^{37}$. En compensation, la Cité universitaire est autorisée à acquérir de l'Imprimerie générale Lahure un terrain de $18188 \mathrm{~m}^{2}$, situé 70, avenue Staline, sur lequel 400 logements devaient être édifiés par le groupe FFF (société de HLM pour fonctionnaires). Ce terrain en forme de hache, acquis le 7 juin 1957 par l'Université de Paris (fig. $\mathbf{n}^{\circ} \mathbf{1 9}$ ), est affecté à la réalisation d'un stade utilisé à partir de 1965 par les résidents de la Cité universitaire et le service académique de la Jeunesse et des Sports. Il s'agrandit en 1968 d'une parcelle de $2500 \mathrm{~m}^{2}$, dite parcelle Clément, expropriée au profit de l'État ${ }^{38}$. 
Figure 18

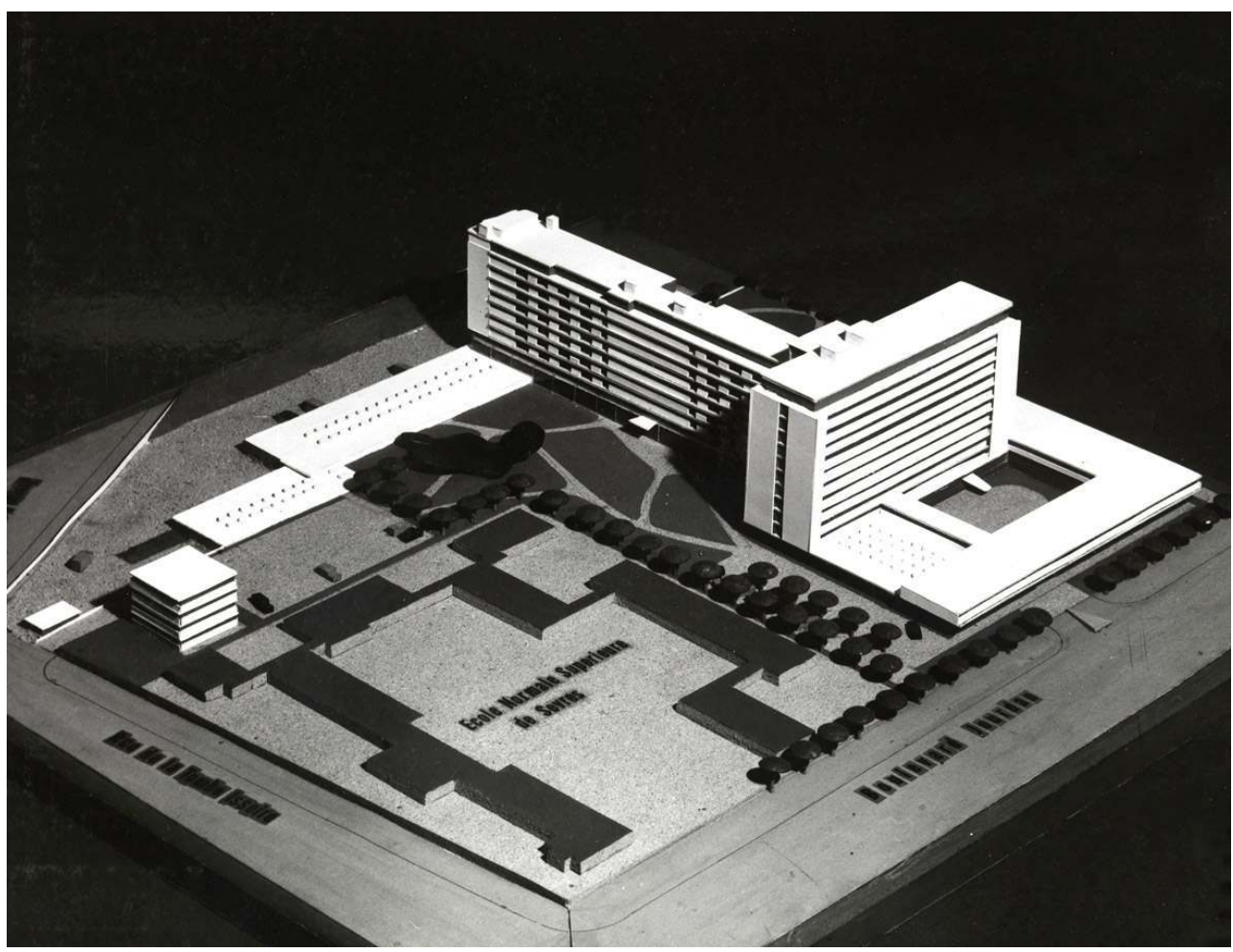

Maquette de l'hôpital de la Cité universitaire construit entre 1958 et 1965 par l'architecte Urbain Cassan sur le terrain de l'ancien dépôt de remonte, à côté de l'ENS de Sèvres. AN, AJ 16/7034. Repro. Ayrault, Philippe.

(c) Conseil régional d'île-de-France, service Patrimoines et Inventaire, 2009. [Base Mémoire : 20097501878NUC4A] 


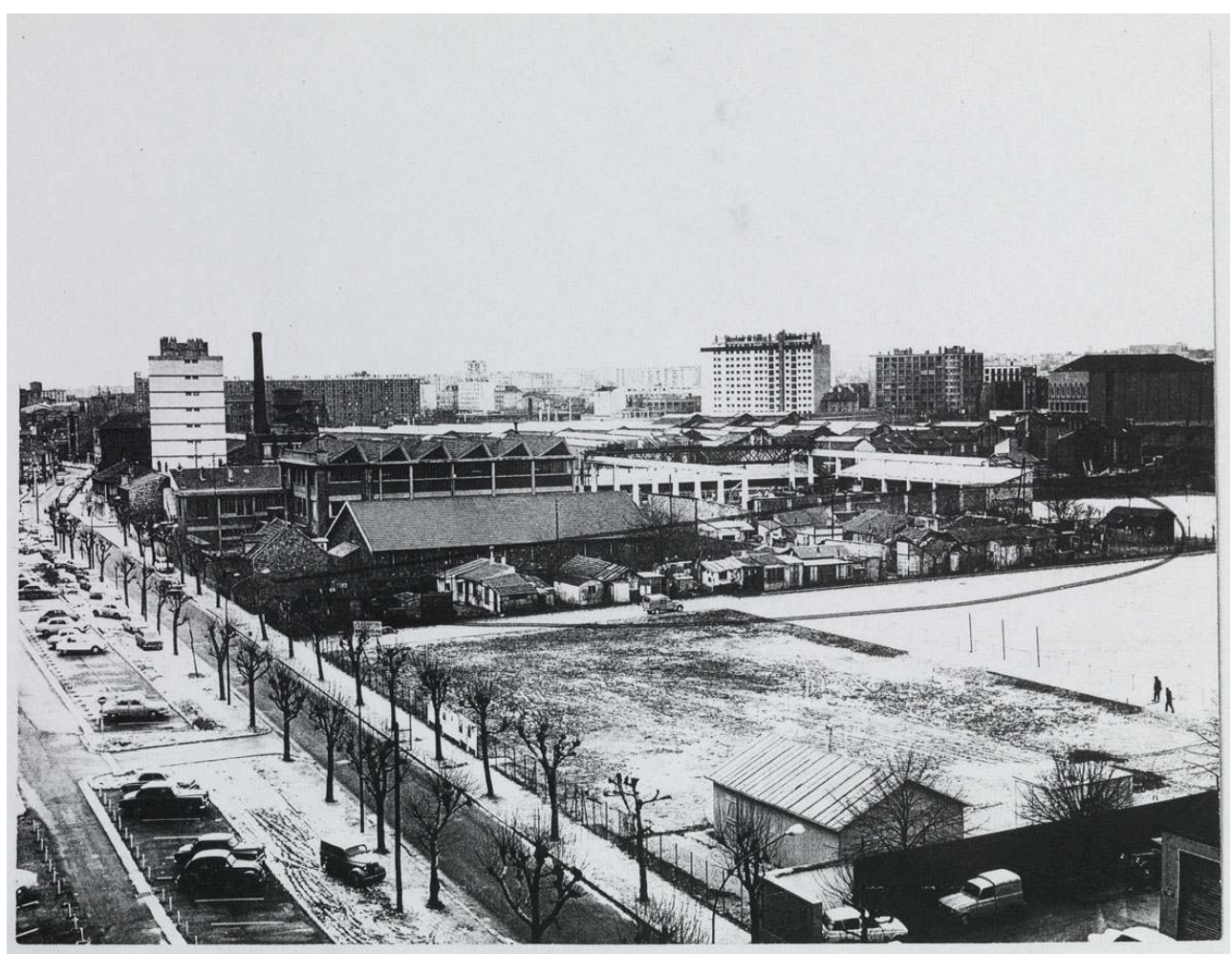

Vue des terrains du Chaperon Vert, le long de la rue Joseph-Staline : la parcelle Lahure acquise en 1957, à l'avant des baraques qui occupent encore la parcelle Clément expropriée en 1968. CAC, 20090013/244. Repro. Kruszyk, Laurent.

(C) Conseil régional d'Île-de-France, service Patrimoines et Inventaire, 2009. [Base Mémoire : 20097500877NUC4A]

\section{Conclusion}

À partir d'une première fondation qui joue le rôle de cellule-mère, la Cité internationale se développe avec une rapidité qui dépasse toutes les prévisions. Dès 1928, ses dirigeants doivent s'assurer la possession de nouveaux espaces libres afin de poursuivre leur tâche sans craindre d'être obligés rapidement d'écarter tout nouveau projet de construction. En 10 ans, trois acquisitions successives permettent d'élargir (d'une douzaine d'hectares) les limites du domaine que la convention de 1921 a mis à leur disposition. Cette phase d'extension s'achève après la seconde guerre mondiale et la création du boulevard périphérique qui ampute la Cité de deux hectares sur sa façade sud. À partir de cette date, et en dépit d'une nouvelle tentative d'agrandissement sur le territoire de Gentilly, la Cité internationale ne peut accueillir de nouvelle résidence qu'en bâtissant à l'intérieur du "périmètre historique» ainsi constitué. Toutefois, une troisième étape de développement s'est amorcée dans les années 2000: engagée depuis 1998 dans un ambitieux programme de rénovation architecturale, la Cité internationale étudie aussi des projets de construction ou de réhabilitation «hors les murs ", à la périphérie de la capitale $^{39}$; la résidence Lila, inaugurée en 2006 dans le $19^{\mathrm{e}}$ arrondissement de Paris, et celle du Quai de Loire, implantée dans l'un des anciens Magasins généraux de la Ville de 
Paris, constituent les deux premières résidences édifiées en dehors du site d'origine du boulevard Jourdan.

\section{NOTES}

1. - BLANCHON, Bernadette. La Cité universitaire de Paris, 1919-1940, mémoire de l'École d'architecture Paris-Villemin, 1991. LEMOINE, Bertrand. La Cité internationale universitaire de Paris. Paris : Hervas, 1990. La Cité internationale universitaire de Paris. Architectures paysagées. Paris: L'Oeil d'or, 2010. Cité internationale universitaire de Paris. Étude historique et documentaire. Bilan foncier et Domanial. GRAHAL, groupe de recherche Art, Histoire, Architecture et Littératures, 2002. Cet article résulte d'une enquête d'inventaire en cours sur le patrimoine architectural et mobilier de la CIUP. http://www.iledefrance.fr/patrimoines-etinventaire

2. - AN, AJ 16/7032, lettre de la Fondation nationale au recteur de l'Université de Paris, 10 mars 1927.

3. - Reconnue d'utilité publique le 6 juin 1925, la Fondation nationale pour le développement de la Cité universitaire de Paris, présidée par André Honnorat, a reçu pour mandat de gérer la Cité universitaire au nom de l'Université de Paris.

4. - D. David-Weill est membre du comité de direction de la Fondation nationale.

5. - Centre des archives contemporaines (CAC), fonds de la Cité internationale universitaire de Paris, 20090013/248, lettre du préfet de la Seine au président de la Fondation nationale, 28 juin 1933.

6. - CAC, 20090013/248, lettre de la Fondation nationale au ministre de l'Éducation nationale, 20 juin 1934.

7. - CAC, 20090013/248, lettre de la Fondation nationale au préfet de la Seine, 15 juin 1934.

8. - Sur ce terrain, seront édifiés la maison du Maroc et le pavillon Walter, la maison de l'Italie et celle des Industries agricoles et alimentaires.

9. - AN, AJ 16/7032, lettre du recteur au ministre de l'Instruction publique, 23 août 1927 ; CAC, 20090013/85, comité de direction de la Fondation nationale du 14 octobre 1927.

10. - CAC, 20090013/250, «Renseignements concernant les terrains de l'ancienne école de dressage », s.d.

11. - Il remplace celui du 27 novembre 1929 qui avait affecté au ministère de l'Instruction publique la totalité du dépôt de remonte, y compris la parcelle de $5865 \mathrm{~m} 2$ à incorporer à la voirie pour l'élargissement du boulevard Jourdan. CAC, 20090013/250, lettre du ministre à A. Honnorat, 26 avril 1930.

12. - CAC, 20090013/236, lettre de la Fondation nationale au préfet de la Seine, 13 février 1931. Par une convention du 16 mai 1947, une partie du terrain de remonte est mise à la disposition de l'École normale supérieure de Sèvres qui y fait édifier des locaux provisoires ; le 8 décembre 1982, cet ensemble immobilier domanial lui est attribué à titre de dotation par arrêté du ministre de l'Économie et des Finances. Les terrains du centre d'hébergement, une fois libérés, ont accueilli des équipements sportifs (par convention du 13 novembre 1935 entre la Cité universitaire et le sous-secrétariat d'État à l'Éducation physique, ils sont mis à la disposition de l'École normale d'Éducation physique) avant d'être affectés en 1955 à la construction de l'hôpital international universitaire (dont la Cité se sépare en 1991). 
13. - La Fondation nationale demande alors au ministère des Finances de faire figurer au budget de l'Instruction publique un crédit lui permettant d'acquérir deux terrains militaires déclassés situés sur la rive gauche de la Seine: champ de manœuvres d'Ivry et parc à fourrages de Vaugirard, de façon à pouvoir entreprendre dans les meilleures conditions des négociations avec la Ville en vue de l'expropriation des terrains de Gentilly. CAC, 20090013/250, lettre de la Fondation nationale au président du Conseil, ministre des Finances, 18 juin 1928.

14. - CAC, 20090013/237. Ministère de l'Instruction publique, exercice 1930-1931, «Subventions diverses pour œuvres de toutes natures en faveur des étudiants ».

15. - CAC, 20090013/85, procès-verbal du comité de direction de la Fondation nationale du 10 juillet 1930. L'arrêté d'affectation du 8 juin 1932 faisant suite à la loi évalue les terrains à 22745 $400 \mathrm{~F}$.

16. - CAC, 20090013/85, procès-verbal du comité de direction de la Fondation nationale du 14 mars 1932

17. - CAC, 20090013/85, Fondation nationale, rapport d'activité de l'année 1933.

18. - Dès le 12 juillet 1928, le comité de direction de la Fondation nationale se demandait, dans une lettre au préfet de la Seine, "s'il n'y aurait pas lieu de profiter de l'obligation dans laquelle va se trouver la Ville de Paris d'effectuer des travaux sur ces terrains pour qu'elle demande à étendre son territoire jusqu'à leur limite ». CAC, 20090013/237.

19. - AD Paris, 2276 W 247, Bulletin municipal officiel de la Ville de Paris du 4 mai 1937.

20. - AD Paris, 2276 W 247, « Mémoire du préfet de la Seine au conseil municipal de la Ville de Paris, sur le nouvel aménagement de voies publiques aux abords de la Cité universitaire avec échange de terrains entre l'État et la Ville de Paris. Projet de rattachement au territoire de Paris d'une partie de ces terrains ", 23 décembre 1936.

21. - À l'ouest du parc de la Cité, création d'une voie promenade de $43 \mathrm{~m}$ de largeur dans le prolongement des avenues encadrant les aqueducs de la Vanne et du Loing; au sud, élargissement à $20 \mathrm{~m}$ de la rue de Montrouge ; ouverture d'une voie biaise de $20 \mathrm{~m}$ de largeur, de cette rue à l'avenue latérale aux aqueducs; élargissement à $15 \mathrm{~m}$ d'un tronçon de la rue du Docteur-Durand.

22. - Une parcelle de $525 \mathrm{~m} 2$ à incorporer aux réservoirs de la Ville, deux terrains de forme triangulaire dont l'un de $1800 \mathrm{~m} 2$ entre la rue du Docteur-Durand, la voie biaise à ouvrir et la rue de Montrouge.

23. - Il souhaite toutefois profiter de cet échange de terrains pour régler à l'amiable deux litiges domaniaux restés en suspens entre la Ville de Paris et l'État concernant l'École polytechnique (rue Descartes) et le Cercle militaire (rue Portalis). CAC, 20090013/243.

24. - CAC, 20090013/243, lettre du ministre des Finances au préfet de la Seine, 7 juin 1937.

25. - Soit une surface constructible d'environ 1 ha 90 et 1 ha 25 de terrains zoniers en location.

26. - Aux termes de ce projet, la Cité universitaire abandonnait à la Ville de Paris une parcelle de terrain de $20400 \mathrm{~m} 2$ (dont $15350 \mathrm{~m} 2$ dépendant anciennement de la commune de Gentilly), et renonçait à la location d'une parcelle zonière de $10250 \mathrm{~m} 2$. En contrepartie, la Ville cédait à la Cité le terrain d'assiette de la rue Alexandre-de-Humboldt (soit $2800 \mathrm{~m} 2$ ) ainsi que la parcelle de $1400 \mathrm{~m} 2$ située près du réservoir de la Vanne. La Ville accordait en outre à la Cité la location d'un terrain zonier de $13900 \mathrm{~m} 2$, situé entre la rue Benoît-Malon et l'avenue de la Porte de Gentilly.

27. - CAC, 20090013/243, « Procès-verbal de la conférence tenue le 17 avril 1952 dans le cabinet du directeur des services d'architecture et d'urbanisme de la Ville de Paris ».

28. - CAC, 20090013/243, « Procès-verbal de la conférence tenue le 17 avril 1952 dans le cabinet du directeur des services d'architecture et d'urbanisme de la Ville de Paris ».

29. - CAC, 20090013/243, lettre de la direction des services d'architecture et d'urbanisme de la préfecture de la Seine au recteur de l'Université de Paris, 30 juillet 1952.

30. - Dont le déclassement sera prononcé par décret du 18 septembre 1953. 
31. - « Dans ces conditions, la surface totale des terrains cédés à la Ville de Paris par la Cité est de $21297 \mathrm{~m} 2$ et la surface des terrains que la Ville cède à l'Université est de $4965 \mathrm{~m} 2$. Au total, la perte de surface pour la Cité est de : 21297 - $4965=16332 \mathrm{~m} 2$ ». Ce nouveau prélèvement entraîne aussi la transformation des installations sportives situées à l'angle sud-est du domaine, qui est prise en charge par l'administration préfectorale. CAC, 20090013/243, lettre du délégué général au directeur des services techniques de la préfecture de la Seine, 25 juillet 1958.

32. - CAC, 20090013/243, lettre du préfet au délégué général, 10 juillet 1958.

33. - CAC, 20090013/243, lettre du préfet au délégué général, 21 juin 1958.

34. - En 1965, un projet de la direction des Domaines de la Seine conclut au versement à l'État d'une soulte de 3 millions environ, mais comme le précédent, il reste lettre morte. La question de la régularisation est toujours en suspens.

35. - En vue de faciliter l'installation de nouvelles usines sur le seul point de la banlieue ayant échappé jusqu'alors aux emprises de l'industrie.

36. - CAC, 20090013/244, dossier " Terrains du Chaperon Vert », note sur l'extension du domaine de la Cité universitaire, février 1955.

37. - CAC, 20090013/244, lettre du ministère de la Reconstruction et du logement au recteur de l'Université de Paris, 19 août 1955.

38. - En 1987, la mairie de Gentilly se porte candidate à l'achat de ce terrain de sports (68-70, rue Lénine), mais à l'heure actuelle, celui-ci appartient toujours à la Cité internationale universitaire. 39. - De nouvelles constructions pourraient aussi à l'avenir densifier la partie du parc située en bordure du périphérique, rendue constructible par le plan local d'urbanisme (PLU) en 2006.

\section{RÉSUMÉS}

La Cité internationale universitaire de Paris a été créée immédiatement après la première guerre mondiale pour favoriser les échanges et la rencontre entre les étudiants du monde entier. Conformément à la convention conclue en 1921 entre la Ville et l'Université de Paris, elle a été édifiée en bordure du parc Montsouris, sur des terrains situés à l'emplacement des anciennes fortifications de Thiers. Dès 1928, le rythme accéléré des constructions pose avec une certaine acuité le problème de l'extension du domaine. Celle-ci s'effectue par étapes durant l'Entre-deuxguerres, notamment aux dépens de la commune voisine de Gentilly. Mais dès le début des années cinquante, le projet de création du boulevard périphérique marque, pour la Cité internationale, la fin des possibilités d'agrandissement à partir du «site historique » du boulevard Jourdan. Aujourd'hui, la Cité se densifie et se développe « hors les murs ».

The Cité internationale universitaire de Paris was created immediately after the First World War in order to encourage exchanges between university students from around the world. According to the 1921 convention established between the city and the University of Paris, the Cite was built along Montsouris Park, on lots previously occupied by the Thiers fortifications. Starting in 1928, the rapid development of the Cité made it necessary to extend the site. Between the two world wars, segments of the neighboring town of Gentilly were progressively annexed. But in the early 1950s, projects for the creation of a boulevard périphérique - beltway - made further extension impossible. Today, construction continues, both on-site and outside the walls of the historical Cité. 
INDEX

Mots-clés : André Honnorat, boulevard périphérique, Cité internationale universitaire de Paris, David David-Weill, Émile Deutsch de la Meurthe, fortifications, Gentilly, hôpital, Paris, urbanisme, zone non aedificandi

\section{AUTEUR}

BRIGITTE BLANC

Service Patrimoines et Inventaire, Région Île-de-France brigitte.blanc@iledefrance.fr 